\title{
Boundedness of Solutions for a Class of Sublinear Reversible Oscillators with Periodic Forcing
}

\author{
Tingting Zhang and Jianguo Si \\ School of Mathematics, Shandong University, Jinan, Shandong 250100, China \\ Correspondence should be addressed to Jianguo Si; sijgmath@yahoo.com.cn \\ Received 3 February 2013; Accepted 30 April 2013 \\ Academic Editor: Wenchang Sun
}

Copyright (C) 2013 T. Zhang and J. Si. This is an open access article distributed under the Creative Commons Attribution License, which permits unrestricted use, distribution, and reproduction in any medium, provided the original work is properly cited.

We study the boundedness of all solutions for the following differential equation $x^{\prime \prime}+f(x) x^{\prime}+(B+\varepsilon e(t))|x|^{\alpha-1} x=p(t)$, where $f(x), p(t)$ are odd functions, $e(t)$ is an even function, $e(t), p(t)$ are smooth 1-periodic functions, $B$ is a nonzero constant, and $\varepsilon$ is a small parameter. A sufficient and necessary condition for the boundedness of all solutions of the above equation is established. Moreover, the existence of Aubry-Mather sets is obtained as well.

\section{Introduction}

It is well known that the longtime behavior for periodically forced planar systems can be very intricate. For example, there are equations having unbounded solutions but with infinitely many zeros and with nearby unbounded solutions having randomly prescribed number of zeros and also periodic solutions; see [1]. In contrast to such unbounded phenomenon Littlewood [2] suggested to study the boundedness of all the solutions of the following differential equation:

$$
\ddot{x}+g(x)=h(t)
$$

in the following two cases:

(i) superlinear case: $g(x) / x \rightarrow+\infty$ as $x \rightarrow \pm \infty$;

(ii) sublinear case: $\operatorname{sgn}(x) \cdot g(x) \rightarrow+\infty$ and $g(x) / x \rightarrow 0$ as $x \rightarrow \pm \infty$. Later, one calls this subject as Littlewood boundedness problem.

The first result in superlinear case is obtained by Morris [3], who showed that all solutions of

$$
\ddot{x}+2 x^{3}=e(t)
$$

are bounded, where $e(t) \in C^{0}$. Later, a series results in superlinear case were obtained by several authors, see [4-13] and references therein. However, in general, it is harder to study the Lagrange stability of sublinear systems since smoothness of sublinear term is insufficient. There are only a few works in sublinear case so far. In 1999, Küpper and You [14] proved the first result in the study of the equation

$$
\ddot{x}+|x|^{\alpha-1} x=p(t),
$$

where $0<\alpha<1$ and $p(t) \in C^{\infty}(\mathbb{T})$. Later, Liu [15] proved the same result for more general equation

$$
\ddot{x}+g(x)=e(t),
$$

where $g(x) \in C^{6}$ satisfying the sublinear condition (ii) and some inequalities, and $e(t) \in C^{5}(\mathbb{T})$. In 2004, Ortega and Verzini [16] studied the boundedness of (4) in a special case with the variational method. In 2009, Wang [17] gave a sufficient and necessary condition for the boundedness of all solutions for sublinear equation

$$
\ddot{x}+e(t)|x|^{\alpha-1} x=p(t),
$$

where $e(t), p(t) \in C^{5}(\mathbb{T})$.

As is widely known, there is a deep similarity between reversible and Hamiltonian dynamics. Many fundamental results of the Hamiltonian systems possess reversible counterparts. On boundedness problem for sublinear reversible systems, the first results were obtained by Li [18], later, Yang [19], in the study of a sublinear reversible systems

$$
\ddot{x}+f(x) \dot{x}+|x|^{\alpha-1} x=e(t) .
$$


Recently, Wang [20] gave a sufficient and necessary condition for the boundedness of all solutions of the differential equation

$$
\ddot{x}+f(x) g(\dot{x})+\gamma|x|^{\alpha-1} x=p(t)
$$

with $0<\alpha<1, \gamma \neq 0$.

By the discussions about the sublinear Hamiltonian equation (1.3) in [17] motivations, we will study the boundedness of all solutions for a sublinear reversible system like

$$
\ddot{x}+f(x) \dot{x}+(B+\varepsilon e(t))|x|^{\alpha-1} x=p(t),
$$

where $B \neq 0$ and $0<\alpha<1$. Furthermore, we also show that (8) has solutions of Mather type. The results obtained in [1820] can be regarded as corollary of result of this paper.

Remark 1. Using the method of this paper we also can consider the more general equation

$$
\ddot{x}+f(x) g(\dot{x})+(B+\varepsilon e(t))|x|^{\alpha-1} x=p(t)
$$

provided of adding suitable conditions for $g(x)$. For convenience, we only consider the case $g(x) \equiv x$.

Remark 2. Adding the perturbation term $\varepsilon e(t)|x|^{\alpha-1} x$ will lead to a new difficulty for estimating $\left|S\left(\theta T_{0}\right)\right|^{\alpha-1} C\left(\theta T_{0}\right)$ appeared in (86). Fortunately, we can easily verify that $\int_{0}^{1}\left|S\left(\theta T_{0}\right)\right|^{\alpha-1} C\left(\theta T_{0}\right) d \theta$ is bounded by a constant (see in the proof of Lemma 12).

Throughout this paper, we denote two universal positive constants without regarding their values by $c<1$ and $C \geq 1$, and suppose that the following conditions hold:

(A1) $f(x) \in C^{4}(\mathbb{R}), p(t) \in C^{3}(\mathbb{T})$ and $e(t) \in C^{3}(\mathbb{\mathbb { T }}), f(x)$ and $p(t)$ are odd, $e(t)$ is even, and $e(t), p(t)$ are both 1 -periodic functions, $\mathbb{T}=\mathbb{R} / \mathbb{Z}$;

(A2) there is some positive constant $\mu$ such that the inequalities

$$
\left|x^{i+1} f^{(i)}(x)\right| \leq C|x|^{\alpha / 2-\beta}
$$

are satisfied for $0 \leq i \leq 4$ and all $|x| \geq \mu$, where $0<$ $\beta<\alpha / 2$.

We decompose $e(t)$ as $e(t)=\bar{e}+\widehat{e}(t)$, where $\bar{e}$ is the average of $e(t)$ and $\hat{e}(t)$ has zero mean value. That is $\bar{e}=\int_{0}^{1} e(s) d s$ and $\int_{0}^{1} \widehat{e}(s) d s=0$. If we write that $A=B+\varepsilon \bar{e}$, then it is easy to see that $A$ and $B$ have the same sign when $0<\varepsilon<\varepsilon^{*}$ with $0<\varepsilon^{*}<|B / \bar{e}|$.

Now we state the main results of this paper.

Theorem 3. Assume that $B \neq 0$ and (A1)-(A2) hold. Then there exists an $0<\varepsilon^{* *}<\varepsilon^{*}$ such that for any $0<\varepsilon<\varepsilon^{* *}$, every solution of (8) is bounded if and only if $B>0$.

Theorem 4. Under the conditions of Theorem 3, there is an $\varepsilon_{0}>0$ such that, for any $\omega \in\left(n, n+\varepsilon_{0}\right)$, (8) has a solution $\left(x_{\omega}(t), x_{\omega}^{\prime}(t)\right)$ of Mather type with rotation number $\omega$. More precisely: (i) if $\omega=p / q$ is rational, the solutions $\left(x_{\omega}(t+i), x_{\omega}^{\prime}(t+\right.$ $i)), 1 \leq i \leq q-1$, are periodic solutions of period $q$; moreover, in this case

$$
\lim _{\omega \rightarrow n} \min _{t \in \mathbb{R}}\left(\left|x_{\omega}(t)\right|+\left|x_{\omega}^{\prime}(t)\right|\right)=+\infty ;
$$

(ii) if $\omega$ is irrational, the solution $\left(x_{\omega}(t), x_{\omega}^{\prime}(t)\right)$ is either a usual quasi-periodic solution or a generalized one.

We recall that a solution is called generalized quasi-periodic if the closed set

$$
\overline{\left\{x(i), x^{\prime}(i), i \in \mathbb{Z}\right\}}
$$

is a Denjoys minimal set.

\section{Reversible Systems and Action-Angle Variables}

In this section, we will assume that $B>0$ and $A>0$. Firstly, we consider (8) which is equivalent to the following system:

$$
\begin{gathered}
\dot{x}=z+P(t), \\
\dot{z}=-A|x|^{\alpha-1} x-\varepsilon \widehat{e}(t)|x|^{\alpha-1} x-f(x)(z+P(t)),
\end{gathered}
$$

where $P(t)=\int_{0}^{t} p(s) d s$. Then we can obtain that (13) is reversible with respect to the transformation $(x, z) \mapsto(-x, z)$ by (A1).

Lemma 5. There exists a $G$-invariant diffeomorphism $(x, y) \rightarrow$ $(x, z)$ such that $(13)$ is transformed into the following system:

$$
\dot{x}=y+\varepsilon E(t)|x|^{\alpha-1} x+P(t),
$$

$$
\begin{aligned}
\dot{y}= & -A|x|^{\alpha-1} x \\
& -\left[\alpha \varepsilon E(t)|x|^{\alpha-1}+f(x)\right]\left[y+\varepsilon E(t)|x|^{\alpha-1} x+P(t)\right],
\end{aligned}
$$

where $E(t)=-\int_{0}^{t} \hat{e}(s) d s$.

Proof. Introduce a transformation $\Psi$ :

$$
x=x, \quad z=y+U(x, t)
$$

where $U(x, t)$ will be determined later. Under this transformation, the system (13) is transformed into a new system as follows:

$$
\begin{gathered}
\dot{x}=y+U(x, t)+P(t) \\
\dot{y}=-A|x|^{\alpha-1} x-\varepsilon \widehat{e}(t)|x|^{\alpha-1} x \\
-\left(f(x)+\frac{\partial U(x, t)}{\partial x}\right)[y+U(x, t)+P(t)] \\
-\frac{\partial U(x, t)}{\partial t} .
\end{gathered}
$$


Now, we define the function $U(x, t)$ by

$$
-\varepsilon \widehat{e}(t)|x|^{\alpha-1} x-\frac{\partial U(x, t)}{\partial t}=0 .
$$

Since $\int_{0}^{1} \widehat{e}(t) d t=0$, so we can obtain $U(x, t)=\varepsilon E(t)|x|^{\alpha-1} x$. Then the new system can be expressed as in (14) by direct computation.

It is easy to know that $U(-x,-t)=U(x, t)$ by $(\mathrm{A} 1)$, then we can obtain that the transformation $\Psi$ is a $G$-invariant diffeomorphism.

Let us consider the auxiliary system

$$
\begin{gathered}
\dot{x}=y, \\
\dot{y}=-A|x|^{\alpha-1} x,
\end{gathered}
$$

which is a time-independent Hamiltonian system with Hamiltonian

$$
H_{0}(x, y)=\frac{y^{2}}{2}+\frac{A}{\alpha+1}|x|^{\alpha+1} \text {. }
$$

It is easy to see that $H_{0}(x, y)>0,(x, y) \in R^{2} \backslash\{0\}$, $H_{0}(0,0)=0$. Note that each level line $H_{0}(x, y)=h>0$ is a close orbit of system (18), hence, all the solutions of (18) are periodic with period tending to zero as $h$ tends to infinity.

Assume that $(S(t), C(t))$ is the solution of (18) with initial conditions $(S(0), C(0))=(0,1)$, and let $T_{0}>0$ be the minimal period. We can find that $S(t)$ and $C(t)$ satisfy

(i) $S(t) \in C^{2}(R), C(t) \in C^{1}(R)$;

(ii) $(S(-t), C(-t))=(-S(t), C(t)),\left(S\left(t+T_{0}\right), C\left(t+T_{0}\right)\right)=$ $(S(t), C(t))$

(iii) $\dot{S}(t)=C(t), \dot{C}(t)=-A|S(t)|^{\alpha-1} S(t)$;

(iv) $(1 / 2) C^{2}(t)+(A /(\alpha+1))|S(t)|^{\alpha+1}=1 / 2$;

(v) $C\left(T_{0} t\right)=0 \Leftrightarrow t(\bmod (1 / 4))=0$;

(vi) $\left(S\left(T_{0}(1 / 2-t)\right), C\left(T_{0}(1 / 2-t)\right)\right)=\left(S\left(T_{0} t\right),-\mathrm{C}\left(T_{0} t\right)\right)$;

(vii) $S\left(T_{0} t\right)=0 \Leftrightarrow t(\bmod (1 / 2))=0$.

Then we introduce the transformation

$$
\begin{aligned}
\Phi: \mathbb{R}^{+} \times \mathbb{T} & \longrightarrow \mathbb{R}^{2} \backslash\{0\} \\
(\rho, \varphi) & \longmapsto(x, y)
\end{aligned}
$$

which is

$$
\begin{gathered}
x=\rho^{b} S\left(\varphi T_{0}\right), \\
y=\rho^{1-b} C\left(\varphi T_{0}\right),
\end{gathered}
$$

where $b=2 /(3+\alpha)$. It is easy to see that $1 / 2<b<2 / 3$ by $0<\alpha<1$. Since $(S(-t), C(-t))=(-S(t), C(t))$, this transformation is invariant with respect to the involutions $(\rho, \varphi) \mapsto(\rho,-\varphi)$ and $(x, y) \mapsto(-x, y)$, and we can find that the mapping $\Phi$ is a generalized canonical transformation by (iv). In fact,

$$
\begin{aligned}
& \left|\frac{\partial(x, y)}{\partial(\rho, \varphi)}\right| \\
& =\left.\left|A b T_{0}\right| S\left(\varphi T_{0}\right)\right|^{\alpha+1}+(1-b) T_{0} C^{2}\left(\varphi T_{0}\right) \mid \\
& =\left|(1-b) T_{0}-\frac{\alpha+1}{2} b T_{0} C^{2}\left(\varphi T_{0}\right)+(1-b) T_{0} C^{2}\left(\varphi T_{0}\right)\right| \\
& =(1-b) T_{0} \text {, } \\
& \left(\begin{array}{c}
\dot{\rho} \\
\dot{\varphi}
\end{array}\right)=\left(\begin{array}{cc}
-d y_{\varphi} & d x_{\varphi} \\
d y_{\rho} & -d x_{\rho}
\end{array}\right)\left(\begin{array}{c}
\dot{x} \\
\dot{y}
\end{array}\right),
\end{aligned}
$$

where $d=\left((1-b) T_{0}\right)^{-1}$.

Under the transformation $\Phi$, the system (18) is transformed into the simpler form

$$
\dot{\rho}=-\frac{\partial h_{0}}{\partial \varphi}=0, \quad \dot{\varphi}=\frac{\partial h_{0}}{\partial \rho}=\frac{1}{T_{0}} \cdot \rho^{1-2 b},
$$

where $h_{0}(\rho)=\left((2-2 b) T_{0}\right)^{-1} \cdot \rho^{2(1-b)}$.

The original system (13) is transformed into the system

$$
\begin{gathered}
\frac{d \rho}{d t}=l_{1}(\rho, \varphi)+l_{2}(\rho, \varphi, t)+\varepsilon l_{3}(\rho, \varphi, t) \\
+\alpha T_{0}\left|S\left(\varphi T_{0}\right)\right|^{\alpha-1} C\left(\varphi T_{0}\right) \varepsilon l_{4}(\rho, \varphi, t), \\
\frac{d \varphi}{d t}=h_{0}^{\prime}(\rho)+h_{1}(\rho, \varphi)+h_{2}(\rho, \varphi, t)+\varepsilon h_{3}(\rho, \varphi, t),
\end{gathered}
$$

where

$$
\begin{gathered}
l_{1}(\rho, \varphi)=-d T_{0} \rho f\left(\rho^{b} S\left(\varphi T_{0}\right)\right) C^{2}\left(\varphi T_{0}\right) \\
=:-d x_{\varphi} f(x) y, \\
l_{2}(\rho, \varphi, t) \quad \\
=-d T_{0} \varepsilon \rho^{2-2 b} f\left(\rho^{b} S\left(\varphi T_{0}\right)\right) \\
\times\left|S\left(\varphi T_{0}\right)\right|^{\alpha-1} S\left(\varphi T_{0}\right) C\left(\varphi T_{0}\right) E(t) \\
+A d T_{0} \rho^{1-b}\left|S\left(\varphi T_{0}\right)\right|^{\alpha-1} S\left(\varphi T_{0}\right) P(t) \\
-d T_{0} \rho^{b} f\left(\rho^{b} S\left(\varphi T_{0}\right)\right) C\left(\varphi T_{0}\right) P(t) \\
=: d \varepsilon x_{\varphi}|x|^{\alpha-1} x f(x) E(t)-d y_{\varphi} P(t)-d x_{\varphi} f(x) P(t), \\
l_{3}(\rho, \varphi, t)=A d T_{0} \rho^{3-4 b}\left|S\left(\varphi T_{0}\right)\right|^{2 \alpha} E(t) \\
=:-d y_{\varphi}|x|^{\alpha-1} x E(t), \\
l_{4}(\rho, \varphi, t) \\
=-d \rho^{3-4 b} C\left(\varphi T_{0}\right) E(t) \\
-d \varepsilon \rho^{4-6 b}\left|S\left(\varphi T_{0}\right)\right|^{\alpha-1} S\left(\varphi T_{0}\right) E^{2}(t) \\
-d \rho^{2-3 b} P(t) E(t),
\end{gathered}
$$


$h_{1}(\rho, \varphi)=d b f\left(\rho^{b} S\left(\varphi T_{0}\right)\right) C\left(\varphi T_{0}\right) S\left(\varphi T_{0}\right)=: d x_{\rho} f(x) y$, $h_{2}(\rho, \varphi, t)$

$$
\begin{aligned}
= & d b \varepsilon \rho^{1-2 b} f\left(\rho^{b} S\left(\varphi T_{0}\right)\right)\left|S\left(\varphi T_{0}\right)\right|^{\alpha+1} E(t) \\
& +\alpha d b \varepsilon^{2} \rho^{3-6 b}\left|S\left(\varphi T_{0}\right)\right|^{2 \alpha} E^{2}(t) \\
& +d(1-b) \rho^{-b} C\left(\varphi T_{0}\right) P(t) \\
& +d b \rho^{b-1} f\left(\rho^{b} S\left(\varphi T_{0}\right)\right) S\left(\varphi T_{0}\right) P(t) \\
& +\alpha d b \varepsilon \rho^{1-3 b}\left|S\left(\varphi T_{0}\right)\right|^{\alpha-1} S\left(\varphi T_{0}\right) E(t) P(t) \\
= & d \varepsilon x_{\rho}|x|^{\alpha-1} x f(x) E(t)+\alpha d \varepsilon^{2} x_{\rho}|x|^{2 \alpha-2} x E^{2}(t) \\
& +d y_{\rho} P(t)+d x_{\rho} f(x) P(t)+\alpha d \varepsilon x_{\rho}|x|^{\alpha-1} E(t) P(t), \\
h_{3}(\rho, \varphi, t) & \\
= & d(1-b+\alpha b) \rho^{2-4 b}\left|S\left(\varphi T_{0}\right)\right|^{\alpha-1} S\left(\varphi T_{0}\right) C\left(\varphi T_{0}\right) E(t) \\
=: & d y_{\rho}|x|^{\alpha-1} x E(t)+\alpha d x_{\rho}|x|^{\alpha-1} y E(t) .
\end{aligned}
$$

Let

$$
\begin{aligned}
& L_{2}(\rho, \varphi, t)= l_{2}(\rho, \varphi, t)+\varepsilon l_{3}(\rho, \varphi, t) \\
&+\alpha T_{0}\left|S\left(\varphi T_{0}\right)\right|^{\alpha-1} C\left(\varphi T_{0}\right) \varepsilon l_{4}(\rho, \varphi, t), \\
& H_{2}(\rho, \varphi, t)=h_{2}(\rho, \varphi, t)+\varepsilon h_{3}(\rho, \varphi, t) .
\end{aligned}
$$

Clearly, $x$ is odd in $\varphi$ and $y$ is even in $\varphi$ by the definitions of $S(t)$ and $C(t)$. Thus, by the evenness of $P(t)$ and the oddness of $f(x)$ and $E(t)$ we have

$$
\begin{array}{ll}
l_{1}(\rho,-\varphi)=-l_{1}(\rho, \varphi), & L_{2}(\rho,-\varphi,-t)=-L_{2}(\rho, \varphi, t), \\
h_{1}(\rho,-\varphi)=h_{1}(\rho, \varphi), & H_{2}(\rho,-\varphi,-t)=H_{2}(\rho, \varphi, t) .
\end{array}
$$

This implies that system (24) is reversible with respect to the involutions $(\rho, \varphi) \mapsto(\rho,-\varphi)$.

Lemma 6. For $0 \leq k+m \leq 4$, the following inequalities hold:

(1) $\left|\left(\partial^{k} / \partial \rho^{k}\right) l_{1}(\rho, \varphi)\right| \leq C \rho^{-k+2-\gamma-(5 / 2) b}$,

(2) $\left|\left(\partial^{k+m} / \partial \rho^{k} \partial t^{m}\right) l_{2}(\rho, \varphi, t)\right| \leq C \rho^{-k+a}$,

(3) $\left|\left(\partial^{k+m} / \partial \rho^{k} \partial t^{m}\right) l_{3}(\rho, \varphi, t)\right| \leq C \rho^{-k+3-4 b}$,

(4) $\left|\left(\partial^{k+m} / \partial \rho^{k} \partial t^{m}\right) l_{4}(\rho, \varphi, t)\right| \leq C \rho^{-k+3-4 b}$,

(5) $\left|\left(\partial^{k} / \partial \rho^{k}\right) h_{1}(\rho, \varphi)\right| \leq C \rho^{-k+1-\gamma-(5 / 2) b}$,

(6) $\left|\left(\partial^{k+m} / \partial \rho^{k} \partial t^{m}\right) h_{2}(\rho, \varphi, t)\right| \leq C \rho^{-k+\tau}$,

(7) $\left|\left(\partial^{k+m} / \partial \rho^{k} \partial t^{m}\right) h_{3}(\rho, \varphi, t)\right| \leq C \rho^{-k+2-4 b}$,

where $\gamma=\beta b, a=\max (3-(9 / 2) b-\gamma, 1-b)$, and $\tau=\max (3-$ $6 b,-b)$.
Proof. (1) It is easy to know that $\left(\partial^{k} / \partial \rho^{k}\right) l_{1}(\rho, \varphi)$ is a sum of terms of the form

$$
d \frac{\partial^{i_{1}} x_{\varphi}}{\partial \rho^{i_{1}}} \cdot \frac{\partial^{i_{2}} f(x)}{\partial \rho^{i_{2}}} \cdot \frac{\partial^{i_{3}} y}{\partial \rho^{i_{3}}}, \quad i_{1}+i_{2}+i_{3}=k,
$$

where $0 \leq i_{1}, i_{2}, i_{3} \leq k$. Meanwhile, $\partial^{i_{2}} f(x) / \partial \rho^{i_{2}}$ is a sum terms of the form

$$
f^{(s)}(x) \cdot \frac{\partial^{l_{1}} x}{\partial \rho^{l_{1}}} \frac{\partial^{l_{2}} x}{\partial \rho^{l_{2}}} \cdots \frac{\partial^{l_{s}} x}{\partial \rho^{l_{s}}}, \quad 0 \leq s \leq i_{2}, l_{1}+\cdots+l_{s}=i_{2} .
$$

Hence, we obtain

$$
\begin{aligned}
\left|\frac{\partial^{k}}{\partial \rho^{k}} l_{1}(\rho, \varphi)\right| & \leq C\left|\rho^{-i_{1}} x \cdot \rho^{-i_{2}} f(x) \cdot \rho^{-i_{3}} y\right| \\
& \leq C \rho^{-k} \cdot|x \cdot f(x) \cdot y| \leq C \rho^{-k}|x|^{\alpha / 2-\beta}|y| \\
& \leq C \rho^{-k+2-\gamma-(5 / 2) b}
\end{aligned}
$$

by the assumptions on $f(x)$ and the definitions of $x(\rho, \varphi)$ and $y(\rho, \varphi)$.

(2) From the expression of $l_{2}(\rho, \varphi, t)$, we have

$$
\begin{gathered}
\left|\frac{\partial^{k+m}\left(-d \varepsilon x_{\varphi}|x|^{\alpha-1} x f(x) E(t)\right)}{\partial \rho^{k} \partial t^{m}}\right| \\
\leq C\left|\frac{\partial^{k}\left(-d \varepsilon x_{\varphi}|x|^{\alpha-1} x f(x)\right)}{\partial \rho^{k}}\right|\left|E^{(m)}(t)\right| \\
\leq C \varepsilon\left|\rho^{-i_{1}} x \cdot \rho^{-i_{2}} f(x) \cdot \rho^{\alpha b-i_{3}}\right| \\
\leq C \varepsilon \rho^{-k+\alpha b}|x \cdot f(x)| \\
\leq C \varepsilon \rho^{-k+3-\gamma-9 b / 2}, \\
\left|\begin{array}{c}
\left|\frac{\partial^{k+m}\left(-d y_{\varphi} P(t)\right)}{\partial \rho^{k} \partial t^{m}}\right| \leq C\left|\frac{\partial^{k}\left(d y_{\varphi}\right)}{\partial \rho^{k}}\right|\left|\frac{d^{m} P(t)}{d t^{m}}\right| \\
\leq C \rho^{-k+1-b}, \\
\leq C \rho^{-k+1-\gamma-3 b / 2} .
\end{array}\right| \frac{\partial^{k+m}\left(-d x_{\varphi} f(x) P(t)\right)}{\partial \rho^{k} \partial t^{m}}|\leq C| \frac{\partial^{k}\left(-d x_{\varphi} f(x)\right)}{\partial \rho^{k}}\left|\frac{d^{m} P(t)}{d t^{m}}\right|
\end{gathered}
$$

We can find that

$$
\left|\frac{\partial^{k+m}}{\partial \rho^{k} \partial t^{m}} l_{2}(\rho, \varphi, t)\right| \leq C \rho^{-k+a},
$$

where $a=\max (3-9 b / 2-\gamma, 1-b)$. 
(3) From the expression of $l_{3}(\rho, \varphi, t)$, we have

$$
\begin{aligned}
& \left|\frac{\partial^{k+m}\left(d y_{\varphi}|x|^{\alpha-1} x E(t)\right)}{\partial \rho^{k} \partial t^{m}}\right| \\
& \quad \leq C\left|\frac{\partial^{k}\left(d y_{\varphi}|x|^{\alpha-1} x\right)}{\partial \rho^{k}}\right|\left|\frac{d^{m}(E(t))}{d t^{m}}\right| \\
& \leq C \rho^{-k+3-4 b} .
\end{aligned}
$$

(4) From the expression of $l_{4}(\rho, \varphi, t)$, we can obtain that

$$
\begin{aligned}
& \left|\frac{\partial^{k+m} l_{4}(\rho, \varphi, t)}{\partial \rho^{k} \partial t^{m}}\right| \\
& \leq C\left|\frac{\partial^{k}\left(-d \rho^{3-4 b} C\left(\varphi T_{0}\right)\right)}{\partial \rho^{k}}\right| \frac{d^{m}(E(t))}{d t^{m}} \mid \\
& +C\left|\frac{\partial^{k}\left(-d \rho^{2-3 b}\right)}{\partial \rho^{k}}\right|\left|\frac{d^{m}(P(t) E(t))}{d t^{m}}\right| \\
& \quad+C\left|\frac{\partial^{k}\left(-d \varepsilon \rho^{4-6 b}\left|S\left(\varphi T_{0}\right)\right|^{\alpha-1} S\left(\varphi T_{0}\right)\right)}{\partial \rho^{k}}\right|\left|\frac{d^{m}\left(E^{2}(t)\right)}{d t^{m}}\right| \\
& \leq C \rho^{-k+3-4 b} .
\end{aligned}
$$

(5) From the definition of $h_{1}(\rho, \varphi)$, we have

$$
\begin{aligned}
\mid \frac{\partial^{k}}{\partial \rho^{k}} & h_{1}(\rho, \varphi) \mid \\
& \leq C\left|\rho^{-i_{1}-1} x \cdot \rho^{-i_{2}} f(x) \cdot \rho^{-i_{3}} y\right| \\
& \leq C \rho^{-k} \cdot|x \cdot f(x) \cdot y| \leq C \rho^{-k-1}|x|^{\alpha / 2-\beta}|y| \\
& \leq C \rho^{-k+1-\gamma-(5 / 2) b} .
\end{aligned}
$$

(6) From the definition of $h_{2}(\rho, \varphi, t)$, we can obtain

$$
\begin{aligned}
& \left|\frac{\partial^{k+m}\left(d \varepsilon x_{\rho}|x|^{\alpha-1} x f(x) E(t)\right)}{\partial \rho^{k} \partial t^{m}}\right| \\
& \leq C\left|\frac{\partial^{k}\left(d x_{\rho}|x|^{\alpha-1} x f(x)\right)}{\partial \rho^{k}}\right|\left|E^{(m)}(t)\right| \\
& \leq C \varepsilon\left|\rho^{-i_{1}-1} x \cdot \rho^{-i_{2}} f(x) \cdot \rho^{\alpha b-i_{3}}\right| \\
& \leq C \varepsilon \rho^{-k+\alpha b-1}|x \cdot f(x)| \\
& \leq C \varepsilon \rho^{-k+2-\gamma-9 b / 2}, \\
& \left|\frac{\partial^{k+m}\left(\alpha d \varepsilon^{2} x_{\rho}|x|^{2 \alpha-2} x E^{2}(t)\right)}{\partial \rho^{k} \partial t^{m}}\right| \\
& \leq C\left|\frac{\partial^{k}\left(\alpha d \varepsilon^{2} x_{\rho}|x|^{2 \alpha-2} x\right)}{\partial \rho^{k}}\right|\left|\frac{d^{m}\left(E^{2}(t)\right)}{d t^{m}}\right| \\
& \leq C \varepsilon \rho^{-k+3-6 b} \text {, }
\end{aligned}
$$

$$
\begin{gathered}
\left|\frac{\partial^{k+m}\left(-d y_{\rho} P(t)\right)}{\partial \rho^{k} \partial t^{m}}\right| \leq C\left|\frac{\partial^{k}\left(d y_{\rho}\right)}{\partial \rho^{k}}\right|\left|\frac{d^{m} P(t)}{d t^{m}}\right| \leq C \rho^{-k-b}, \\
\left|\frac{\partial^{k+m}\left(d x_{\rho} f(x) P(t)\right)}{\partial \rho^{k} \partial t^{m}}\right| \leq C\left|\frac{\partial^{k}\left(-d x_{\rho} f(x)\right)}{\partial \rho^{k}}\right|\left|\frac{d^{m} P(t)}{d t^{m}}\right| \\
\leq C \rho^{-k-\gamma-3 b / 2}, \\
\left|\frac{\partial^{k+m}\left(\alpha d \varepsilon x_{\rho}|x|^{\alpha-1} E(t) P(t)\right)}{\partial \rho^{k} \partial t^{m}}\right| \\
\leq C\left|\frac{\partial^{k}\left(\alpha d \varepsilon^{2} x_{\rho}|x|^{2 \alpha-2} x\right)}{\partial \rho^{k}}\right|\left|\frac{d^{m}(E(t) P(t))}{d t^{m}}\right| \\
\leq C \varepsilon \rho^{-k+1-3 b} .
\end{gathered}
$$

Hence, we can know that

$$
\left|\frac{\partial^{k+m}}{\partial \rho^{k} \partial t^{m}} h_{2}(\rho, \varphi, t)\right| \leq C \rho^{-k+\tau},
$$

where $\tau=\max (3-6 b,-b)$.

(7) From the expression of $h_{3}(\rho, \varphi, t)$, we have

$$
\begin{aligned}
& \left|\frac{\partial^{k+m}}{\partial \rho^{k} \partial t^{m}} h_{3}(\rho, \varphi, t)\right| \\
& \leq\left|\frac{\partial^{k+m}\left(d(1-b+\alpha b) \rho^{2-4 b}\left|S\left(\varphi T_{0}\right)\right|^{\alpha-1} S\left(\varphi T_{0}\right) C\left(\varphi T_{0}\right) E(t)\right)}{\partial \rho^{k} \partial t^{m}}\right| \\
& \leq C\left|\frac{\partial^{k}\left(d(1-b+\alpha b) \rho^{2-4 b}\left|S\left(\varphi T_{0}\right)\right|^{\alpha-1} S\left(\varphi T_{0}\right) C\left(\varphi T_{0}\right)\right)}{\partial \rho^{k}}\right| \\
& \quad \times\left|\frac{d^{m}(E(t))}{d t^{m}}\right| \\
& \leq C \rho^{-k+2-4 b} .
\end{aligned}
$$

For $\lambda_{0}>0$, we define the domain

$$
\mathscr{A}_{\lambda_{0}}=\left\{(\lambda, \varphi, t): \lambda \geq \lambda_{0},(\varphi, t) \in \mathbb{T}^{2}\right\} .
$$

Lemma 7. There exists a G-invariant diffeomorphism $\Psi_{1}$ :

$$
\rho=I+U_{1}(I, \theta), \quad \varphi=\theta
$$

such that $\mathscr{A}_{I^{+}} \subset \Psi_{1}\left(\mathscr{A}_{I_{0}}\right) \subset \mathscr{A}_{I_{-}}$for some $I_{-}<I_{0}<I_{+}$. Under this transformation, (24) is transformed into the system

$$
\begin{gathered}
\frac{d I}{d t}=\widetilde{l}_{1}(I, \theta)+\widetilde{l}_{2}(I, \theta, t)+\varepsilon \widetilde{l}_{3}(I, \theta, t) \\
+\alpha T_{0}\left|S\left(\theta T_{0}\right)\right|^{\alpha-1} C\left(\theta T_{0}\right) \varepsilon \widetilde{l}_{4}(I, \theta, t), \\
\frac{d \theta}{d t}=h_{0}^{\prime}(I)+\widetilde{h}_{1}(I, \theta)+\widetilde{h}_{2}(I, \theta, t)+\varepsilon \widetilde{h}_{3}(I, \theta, t),
\end{gathered}
$$


where

$$
\begin{gathered}
\tilde{l}_{1}(I, \theta)=\frac{\partial V_{1}(\rho, \varphi)}{\partial \rho} \cdot l_{1}(\rho, \varphi)+\frac{\partial V_{1}(\rho, \varphi)}{\partial \varphi} \cdot h_{1}(\rho, \varphi) \\
\tilde{l}_{2}(I, \theta, t)=l_{2}(\rho, \varphi, t) \\
+\frac{\partial V_{1}(\rho, \varphi)}{\partial \rho} \cdot\left(l_{2}(\rho, \varphi, t)+\varepsilon l_{3}(\rho, \varphi, t)\right) \\
+\frac{\partial V_{1}(\rho, \varphi)}{\partial \varphi} \cdot\left(h_{2}(\rho, \varphi, t)+\varepsilon h_{3}(\rho, \varphi, t)\right) \\
+\varepsilon\left(l_{3}(\rho, \varphi, t)-l_{3}(I, \theta, t)\right), \\
\tilde{l}_{3}(I, \theta, t)=l_{3}(I, \theta, t), \\
\tilde{l}_{4}(I, \theta, t)=l_{4}(\rho, \varphi, t)+\frac{\partial V_{1}(\rho, \varphi)}{\partial \rho} \cdot l_{4}(\rho, \varphi, t), \\
\tilde{h}_{1}(I, \theta)=h_{0}^{\prime}(\rho)-h_{0}^{\prime}(I)+h_{1}(\rho, \varphi), \\
\tilde{h}_{2}(I, \theta, t)=h_{2}(\rho, \varphi, t)+\varepsilon\left(h_{3}(\rho, \varphi, t)-h_{3}(I, \theta, t)\right), \\
\tilde{h}_{3}(I, \theta, t)=h_{3}(I, \theta, t),
\end{gathered}
$$

with

$$
V_{1}(\rho, \varphi)=-\int_{0}^{\varphi} \frac{l_{1}(\rho, s)}{h_{0}^{\prime}(\rho)} d s
$$

Proof. Define a transformation $\Phi_{1}$ by

$$
\Phi_{1}: I=\rho+V_{1}(\rho, \varphi), \quad \theta=\varphi .
$$

By

$$
\begin{gathered}
l_{1}(\rho,-\varphi)=-l_{1}(\rho, \varphi), \\
\left|\frac{\partial^{k}}{\partial \rho^{k}} l_{1}(\rho, \varphi)\right| \leq C \rho^{-k+2-\gamma-(5 / 2) b}, \quad 0 \leq k \leq 4,
\end{gathered}
$$

we get

$$
\begin{aligned}
V_{1}(\rho,-\varphi) & =V_{1}(\rho, \varphi), \\
\left|\frac{\partial^{k}}{\partial \rho^{k}} V_{1}(\rho, \varphi)\right| & \leq C \rho^{-k+1-\gamma-b / 2} .
\end{aligned}
$$

Let $\Psi_{1}=\Phi_{1}^{-1}: \rho=I+U_{1}(I, \theta), \varphi=\theta$. The system (24) is transformed into (41).

Lemma 8. For I large enough, the following conclusions hold:

(i) $\left|\partial^{k} U_{1}(I, \theta) / \partial I^{k}\right| \leq C I^{-k+1-\gamma-b / 2}$,

(ii) $U_{1}(I,-\theta)=U_{1}(I, \theta)$.

Proof. In view of

$$
I=\rho+V_{1}(\rho, \varphi), \quad \rho=I+U_{1}(I, \theta),
$$

we obtain

$$
U_{1}(I, \theta)=-V_{1}\left(I+U_{1}(I, \theta), \theta\right) .
$$

By $\left|\left(\partial^{k} / \partial \rho^{k}\right) V_{1}(\rho, \varphi)\right| \leq C \rho^{-k+1-\gamma-b / 2}$, we have $\mid(\partial / \partial \rho) V_{1}(\rho$, $\varphi) \mid \leq C \rho^{-\gamma-b / 2} \leq 1 / 2$ for $\rho$ large enough. Hence, $U_{1}$ is uniquely determined by the contraction mapping principle. Moreover, $U_{1}(\cdot, \theta) \in C^{\infty}\left(\mathscr{A}_{I_{0}}\right)$, for some $I_{0}>0$, as a consequence of the implicit function theorem and

$$
I^{-(1-\gamma-b / 2)}\left|U_{1}(I, \theta)\right| \leq C
$$

Above all, if $k=1$, from (47) and (49), we get

$$
\begin{aligned}
\left|\frac{\partial U_{1}}{\partial I}\right| & =\left|\frac{\partial V_{1} / \partial \rho}{1+\partial V_{1} / \partial \rho}\right| \leq \sum_{n=0}^{\infty}\left(C \rho^{-1+1-\gamma-b / 2}\right)^{n+1} \\
& \leq C \cdot \rho^{-1+1-\gamma-b / 2} \\
& =C \cdot I^{-1+1-\gamma-b / 2}\left(1+\frac{U_{1}}{I}\right)^{-1+1-\gamma-b / 2} \\
& \leq C \cdot I^{-1+1-\gamma-b / 2} .
\end{aligned}
$$

We note that

$$
\frac{\partial^{k} U_{1}(I, \theta)}{\partial I^{k}}=\frac{\partial^{k} V_{1}\left(I+U_{1}(I, \theta), \theta\right)}{\partial I^{k}},
$$

and the right side hand is sum of the term

$$
\frac{\partial^{s} V_{1}}{\partial \rho^{s}} \cdot \frac{\partial^{k_{1}}\left(I+U_{1}\right)}{\partial I^{k_{1}}} \cdots \frac{\partial^{k_{s}}\left(I+U_{1}\right)}{\partial I^{k_{s}}},
$$

where $1 \leq s \leq k, k_{1}+\cdots+k_{s}=k, k_{i} \geq 1$ (for $1 \leq$ $i \leq s)$. The highest order term in $U_{1}$ is the one with $s=1$, namely, $\left(\partial V_{1} / \partial \rho\right) \cdot\left(\partial^{k}\left(I+U_{1}\right) / \partial I^{k}\right)$. We move the part $\left(\partial V_{1} / \partial \rho\right) \cdot\left(\partial^{k} U_{1} / \partial I^{k}\right)$ to the left hand side of (52). Since $\left|(\partial / \partial \rho) V_{1}(\rho, \varphi)\right| \leq 1 / 2$ for $\rho$ large enough, this also provides immediately a bound on $\partial^{k} U_{1}(I, \theta) / \partial I^{k}$. The rest part $\left|\left(\partial V_{1} / \partial \rho\right) \cdot\left(\partial^{k} I / \partial I^{k}\right)\right| \leq C I^{-k+1-\gamma-b / 2}$.

Now, we proceed inductively by assuming that for $j \leq k-$ 1 the estimates

$$
\left|\frac{\partial^{j} U_{1}(I, \theta)}{\partial I^{j}}\right| \leq C I^{-j+1-\gamma-b / 2}
$$

hold and we wish to conclude that the same estimate holds for $j=k$.

Indeed, if $s \geq 2$, we have

$$
\begin{aligned}
& \mid \frac{\partial^{s} V_{1}}{\partial \rho^{s}} \\
& \cdot \frac{\partial^{k_{1}}\left(I+U_{1}\right)}{\partial I^{k_{1}}} \cdots \frac{\partial^{k_{s}}\left(I+U_{1}\right)}{\partial I^{k_{s}}} \mid \\
& \quad \leq C \cdot\left(I+U_{1}\right)^{-s+1-\gamma-b / 2} \cdot I^{-k_{1}+1} \cdots I^{-k_{s}+1} \\
& \quad \leq C \cdot I^{-k+1-\gamma-b / 2}
\end{aligned}
$$

by

$$
\left|\frac{\partial^{j}\left(I+U_{1}(I, \theta)\right)}{\partial I^{j}}\right| \leq C I^{-j+1}, \quad 1 \leq j \leq k-1 .
$$

This proves (i) of Lemma 8. 
Now we check (ii). In fact, since

$$
\begin{gathered}
U_{1}(I, \theta)=-V_{1}\left(I+U_{1}(I, \theta), \theta\right), \\
U_{1}(I,-\theta)=-V_{1}\left(I+U_{1}(I,-\theta), \theta\right),
\end{gathered}
$$

we have

$$
\left|U_{1}(I, \theta)-U_{1}(I,-\theta)\right| \leq \sup _{I \geq I_{0}}\left|\frac{\partial V_{1}}{\partial \rho}\right|\left|U_{1}(I, \theta)-U_{1}(I,-\theta)\right| .
$$

From (47), we have $\left|(\partial / \partial \rho) V_{1}(\rho, \varphi)\right| \leq 1 / 2$ for $I \geq I_{0}$ sufficiently large and therefore we obtain $U_{1}(I, \theta)=U_{1}(I,-\theta)$.

By the estimates in Lemma 6, we can prove the following inequalities.

Lemma 9. For $0 \leq k+m \leq 4$, the following inequalities hold:

(1) $\left|\left(\partial^{k} / \partial I^{k}\right) \widetilde{l}_{1}(I, \theta)\right| \leq C I^{-k+2-2 \gamma-3 b}$,

(2) $\left|\left(\partial^{k+m} / \partial I^{k} \partial t^{m}\right) \widetilde{l}_{2}(I, \theta, t)\right| \leq C I^{-k+a}$,

(3) $\left|\left(\partial^{k+m} / \partial I^{k} \partial t^{m}\right) \tilde{l}_{3}(I, \theta, t)\right| \leq C I^{-k+3-4 b}$,

(4) $\left|\left(\partial^{k+m} / \partial I^{k} \partial t^{m}\right) \tilde{l}_{4}(I, \theta, t)\right| \leq C I^{-k+3-4 b}$,

(5) $\left|\left(\partial^{k} / \partial I^{k}\right) \widetilde{h}_{1}(I, \theta)\right| \leq C I^{-k+1-\gamma-(5 / 2) b}$,

(6) $\left|\left(\partial^{k+m} / \partial I^{k} \partial t^{m}\right) \widetilde{h}_{2}(I, \theta, t)\right| \leq C I^{-k+\tau}$,

(7) $\left|\left(\partial^{k+m} / \partial I^{k} \partial t^{m}\right) \widetilde{h}_{3}(I, \theta, t)\right| \leq C I^{-k+2-4 b}$.

Proof. (1) From the estimates (1) and (5) of Lemmas 6 and 8, it follows that

$$
\begin{aligned}
& \left|\frac{\partial^{k}}{\partial I^{k}} \tilde{l}_{1}(I, \theta)\right| \\
& \leq\left|\frac{\partial^{k}}{\partial I^{k}}\left(\frac{\partial V_{1}(\rho, \varphi)}{\partial \rho} \cdot l_{1}(\rho, \varphi)\right)\right|+\left|\frac{\partial^{k}}{\partial I^{k}}\left(\frac{\partial V_{1}(\rho, \varphi)}{\partial \varphi} \cdot h_{1}(\rho, \varphi)\right)\right| \\
& \leq C \sum_{i_{1}+i_{2}=k}\left|\frac{\partial^{i_{1}+1} V_{1}(\rho, \varphi)}{\partial I^{i_{1}} \partial \rho}\right|\left|\frac{\partial^{i_{2}} l_{1}}{\partial I^{i_{2}}}\right|+C \sum_{i_{1}+i_{2}+i_{3}=k} \frac{\partial^{i_{1}} \rho^{2 b-1}}{\partial I^{i_{1}}}\left|\frac{\partial^{i_{2}} l_{1}}{\partial I^{i_{2}}}\right|\left|\frac{\partial^{i_{3}} h_{1}}{\partial I^{i_{3}}}\right| \\
& \leq C \sum_{i_{1}+i_{2}=k}\left(\sum_{\tau_{1}+\cdots+\tau_{s}=i_{1}}\left|\frac{\partial^{s+1} V_{1}(\rho, \varphi)}{\partial \rho^{s+1}} \frac{\partial^{\tau_{1}}\left(I+U_{1}\right)}{\partial I^{\tau_{1}}} \cdots \frac{\partial^{\tau_{s}}\left(I+U_{1}\right)}{\partial I^{\tau_{s}}}\right|\right) \\
& \quad \times\left|\frac{\partial^{i_{2}} l_{1}}{\partial I^{i_{2}}}\right| \sum_{i_{1}+i_{2}+i_{3}=k}\left(\sum_{\tau_{1}+\cdots+\tau_{s}=i_{1}}\left|\frac{\partial^{s} \rho^{2 b-1}}{\partial \rho^{s}} \frac{\partial^{\tau_{1}}\left(I+U_{1}\right)}{\partial I^{\tau_{1}}} \cdots \frac{\partial^{\tau_{s}}\left(I+U_{1}\right)}{\partial I^{\tau_{s}}}\right|\right) \\
& \times\left|\frac{\partial^{i_{2}} l_{1}}{\partial I^{i_{2}}} \frac{\partial^{i_{3}} h_{1}}{\partial I^{i_{3}}}\right| \\
& \leq C \rho^{-k+2-2 \gamma-3 b} \leq C I^{-k+2-2 \gamma-3 b} .
\end{aligned}
$$

(2) Since

$$
\begin{aligned}
\tilde{l}_{2}(I, \theta, t)= & l_{2}(\rho, \varphi, t) \\
& +\frac{\partial V_{1}(\rho, \varphi)}{\partial \rho} \cdot\left(l_{2}(\rho, \varphi, t)+\varepsilon l_{3}(\rho, \varphi, t)\right) \\
& +\frac{\partial V_{1}(\rho, \varphi)}{\partial \varphi} \cdot\left(h_{2}(\rho, \varphi, t)+\varepsilon h_{3}(\rho, \varphi, t)\right) \\
& +\varepsilon\left(l_{3}(\rho, \varphi, t)-l_{3}(I, \theta, t)\right),
\end{aligned}
$$

we can prove that

$$
\begin{gathered}
\left|\frac{\partial^{k+m}}{\partial I^{k} \partial t^{m}}\left(\frac{\partial V_{1}(\rho, \varphi)}{\partial \rho} \cdot\left(l_{2}(\rho, \varphi, t)+\varepsilon l_{3}(\rho, \varphi, t)\right)\right)\right| \\
\leq C I^{-k+a}, \\
\left|\frac{\partial^{k+m}}{\partial I^{k} \partial t^{m}}\left(\frac{\partial V_{1}(\rho, \varphi)}{\partial \varphi} \cdot\left(h_{2}(\rho, \varphi, t)+\varepsilon h_{3}(\rho, \varphi, t)\right)\right)\right| \\
\leq C I^{-k+a}, \\
\quad\left|\frac{\partial^{k+m}}{\partial I^{k} \partial t^{m}} l_{2}(\rho, \varphi, t)\right| \leq I^{-k+a} .
\end{gathered}
$$

Their proofs are similar to the proofs in (1).

Next, we check the last part of $\tilde{l}_{2}(I, \theta, t)$. We get

$$
\begin{aligned}
& \left|\frac{\partial^{k+m}}{\partial I^{k} \partial t^{m}}\left(l_{3}(\rho, \varphi, t)-l_{3}(I, \theta, t)\right)\right| \\
& \quad=\left|\frac{\partial^{k+m}}{\partial I^{k} \partial t^{m}}\left(\int_{0}^{1} \frac{\partial l_{3}}{\partial \rho}\left(I+s U_{1}(I, \theta), \theta, t\right) \cdot U_{1}(I, \theta) d s\right)\right| \\
& \quad \leq \int_{0}^{1} \sum_{i_{1}+i_{2}=k}\left|\frac{\partial^{i_{1}+m}}{\partial I^{i_{1}} \partial t^{m}}\left(\frac{\partial l_{3}\left(I+s U_{1}, \theta, t\right)}{\partial \rho}\right)\right|\left|\frac{\partial^{i_{2}} U_{1}}{\partial I^{i_{2}}}\right| d s \\
& \quad \leq C \int_{0}^{1} \sum_{i_{1}+i_{2}=k}\left(I+U_{1}\right)^{-i_{1}+2-4 b} I^{-i_{2}+1-\gamma-b / 2} d s \\
& \quad \leq C I^{-k+3-\gamma-(9 / 2) b} \leq C I^{-k+a},
\end{aligned}
$$

by the estimate in Lemma 6 and the definition of $a$.

(3) It is clearly by (3) in Lemma 6.

(4) It is clearly by (4) in Lemmas 6 and 8.

(5) We have that

$$
\widetilde{h}_{1}(I, \theta)=h_{0}^{\prime}(\rho)-h_{0}^{\prime}(I)+h_{1}(\rho, \varphi),
$$

$$
\left|\frac{\partial^{k+m}}{\partial I^{k} \partial t^{m}}\left(h_{0}^{\prime}(\rho)-h_{0}^{\prime}(I)\right)\right|
$$

$$
\begin{aligned}
& \leq\left|\frac{\partial^{k+m}}{\partial I^{k} \partial t^{m}}\left(\int_{0}^{1} \frac{d^{2} h_{0}\left(I+s U_{1}\right)}{d \rho^{2}} U_{1}(I, \theta) d s\right)\right| \\
& \leq C I^{-k-2 b+1-\gamma-b / 2} \leq C I^{-k+1-\gamma-5 b / 2} .
\end{aligned}
$$


From the last inequalities and (5) in Lemma 6, we obtain

$$
\left|\frac{\partial^{k}}{\partial I^{k}} \widetilde{h}_{1}(I, \theta)\right| \leq C I^{-k+1-\gamma-(5 / 2) b} .
$$

(6) Since

$$
\begin{gathered}
\tilde{h}_{2}(I, \theta, t)=h_{2}(\rho, \varphi, t)+\varepsilon\left(h_{3}(\rho, \varphi, t)-h_{3}(I, \theta, t)\right), \\
\left|\frac{\partial^{k+m}}{\partial I^{k} \partial t^{m}} h_{2}(\rho, \varphi, t)\right| \leq C I^{-k+\tau},
\end{gathered}
$$

we just have to prove that

$$
\left|\frac{\partial^{k+m}}{\partial I^{k} \partial t^{m}}\left(h_{3}(\rho, \varphi, t)-h_{3}(I, \theta, t)\right)\right| \leq C I^{-k+\tau} .
$$

In fact,

$$
\begin{aligned}
& \left|\frac{\partial^{k+m}}{\partial I^{k} \partial t^{m}}\left(h_{3}(\rho, \varphi, t)-h_{3}(I, \theta, t)\right)\right| \\
& \quad=\left|\frac{\partial^{k+m}}{\partial I^{k} \partial t^{m}}\left(\int_{0}^{1} \frac{\partial h_{3}}{\partial \rho}\left(I+s U_{1}(I, \theta), \theta, t\right) \cdot U_{1}(I, \theta) d s\right)\right| \\
& \quad \leq \int_{0}^{1} \sum_{i_{1}+i_{2}=k}\left|\frac{\partial^{i_{1}+m}}{\partial I^{i_{1}} \partial t^{m}}\left(\frac{\partial h_{3}\left(I+s U_{1}, \theta, t\right)}{\partial \rho}\right)\right|\left|\frac{\partial^{i_{2}} U_{1}}{\partial I^{i_{2}}}\right| d s \\
& \leq C \int_{0}^{1} \sum_{i_{1}+i_{2}=k}\left(I+U_{1}\right)^{-i_{1}+1-4 b} I^{-i_{2}+1-\gamma-b / 2} d s \\
& \leq C I^{-k+2-\gamma-(9 / 2) b} \leq C I^{-k+\tau},
\end{aligned}
$$

so we have proved (6)

(7) We have

$$
\left|\frac{\partial^{k+m}}{\partial I^{k} \partial t^{m}} \widetilde{h}_{3}(I, \theta, t)\right| \leq C I^{-k+2-4 b}
$$

by (7) in Lemma 6.

\section{The Proof of Boundedness}

In this section, all the solutions of (8) which are bounded will be proved via the KAM theory for reversible systems developed by Sevryuk [21] or Moser [22, 23] if $B>0$.

We define the functions $\eta_{0}, \eta_{1}, \eta_{2}, \eta_{3}, \xi_{1}, \xi_{2}$, and $\xi_{3}$ as

$$
\begin{gathered}
\eta_{0}(I)=\frac{1}{h_{0}^{\prime}(I)}, \\
\eta_{1}(I, \theta)=-\frac{\widetilde{h}_{1}(I, \theta)}{h_{0}^{\prime}(I)\left(h_{0}^{\prime}(I)+\widetilde{h}_{1}(I, \theta)\right)},
\end{gathered}
$$

$\eta_{2}(I, \theta, t)$

$$
\begin{aligned}
= & -\left(\widetilde{h}_{2}(I, \theta, t)\right) \\
& \times\left(\left(h_{0}^{\prime}(I)+\widetilde{h}_{1}(I, \theta)\right)\right. \\
& \left.\quad \times\left(h_{0}^{\prime}(I)+\widetilde{h}_{1}(I, \theta)+\widetilde{h}_{2}(I, \theta, t)+\varepsilon \widetilde{h}_{3}(I, \theta, t)\right)\right)^{-1},
\end{aligned}
$$

$$
\begin{aligned}
& \eta_{3}(I, \theta, t) \\
&=-\left(\widetilde{h}_{3}(I, \theta, t)\right) \\
& \times\left(\left(h_{0}^{\prime}(I)+\widetilde{h}_{1}(I, \theta)\right)\right. \\
&\left.\times\left(h_{0}^{\prime}(I)+\widetilde{h}_{1}(I, \theta)+\widetilde{h}_{2}(I, \theta, t)+\varepsilon \widetilde{h}_{3}(I, \theta, t)\right)\right)^{-1}, \\
& \xi_{1}(I, \theta, t)=\left(\tilde{l}_{1}(I, \theta)+\tilde{l}_{2}(I, \theta, t)\right) \\
& \cdot\left(\eta_{0}(I)+\eta_{1}(I, \theta)+\eta_{2}(I, \theta, t)+\varepsilon \eta_{3}(I, \theta, t)\right), \\
& \xi_{2}(I, \theta, t)=\widetilde{l}_{3}(I, \theta, t) \\
& \cdot\left(\eta_{0}(I)+\eta_{1}(I, \theta)+\eta_{2}(I, \theta, t)+\varepsilon \eta_{3}(I, \theta, t)\right), \\
& \tilde{l}_{4}(I, \theta, t) \\
& \cdot\left(\eta_{0}(I)+\eta_{1}(I, \theta)+\eta_{2}(I, \theta, t)+\varepsilon \eta_{3}(I, \theta, t)\right) .
\end{aligned}
$$

Then system (41) is equivalent to the following system:

$$
\begin{gathered}
\frac{d t}{d \theta}=\eta_{0}(I)+\eta_{1}(I, \theta)+\eta_{2}(I, \theta, t)+\varepsilon \eta_{3}(I, \theta, t), \\
\frac{d I}{d \theta}=\xi_{1}(I, \theta, t)+\varepsilon \xi_{2}(I, \theta, t) \\
+\alpha T_{0}\left|S\left(\theta T_{0}\right)\right|^{\alpha-1} C\left(\theta T_{0}\right) \varepsilon \xi_{3}(I, \theta, t) .
\end{gathered}
$$

In addition, one can verify that system (70) is reversible with respect to involution $G:(t, I) \mapsto(-t, I)$.

Then some estimates on the functions $\eta_{i}(i=0,1,2,3)$ and $\xi_{i}(i=1,2,3)$ are given.

Lemma 10. The following inequalities hold:

(1) $c I^{2 b-1} \leq\left|\eta_{0}(I)\right| \leq C I^{2 b-1}$,

(2) $\left|\left(\partial^{k} / \partial I^{k}\right) \eta_{1}(I, \theta)\right| \leq C I^{-k-1-\gamma+3 b / 2}$,

(3) $\left|\left(\partial^{k+m} / \partial I^{k} \partial t^{m}\right) \eta_{2}(I, \theta, t)\right| \leq C I^{-k+\tau+4 b-2}$,

(4) $\left|\left(\partial^{k+m} / \partial I^{k} \partial t^{m}\right) \eta_{3}(I, \theta, t)\right| \leq C I^{-k}$,

(5) $\left|\left(\partial^{k+m} / \partial I^{k} \partial t^{m}\right) \xi_{1}(I, \theta, t)\right| \leq C I^{-k+a+2 b-1}$,

(6) $\left|\left(\partial^{k+m} / \partial I^{k} \partial t^{m}\right) \xi_{2}(I, \theta, t)\right| \leq C I^{-k+2-2 b}$,

(7) $\left|\left(\partial^{k+m} / \partial I^{k} \partial t^{m}\right) \xi_{3}(I, \theta, t)\right| \leq C I^{-k+2-2 b}$, for $0 \leq k+m \leq$ 4.

Proof. (1) It is clear.

(2) Note that $1-2 b>1-\gamma-2 b / 5$, and

$$
\left|\widetilde{h}_{1}(I, \theta)\right| \leq C I^{1-\gamma-(5 / 2) b},
$$


it follows that

$$
\begin{aligned}
\left|h_{0}^{\prime}(I)+\widetilde{h}_{1}(I, \theta)\right| & \geq|| h_{0}^{\prime}(I)|-| \widetilde{h}_{1}(I, \theta)|| \\
& \geq\left|h_{0}^{\prime}(I)\right|-\left|\widetilde{h}_{1}(I, \theta)\right| \\
& \geq \frac{1}{T_{0}} I^{1-2 b}-C I^{1-\gamma-(5 / 2) b} \\
& \geq c I^{1-2 b}
\end{aligned}
$$

as $I \gg 1$.

Moreover, we also have

$$
\begin{aligned}
\left|\frac{\partial^{l}}{\partial I^{l}}\left(h_{0}^{\prime}(I)+\widetilde{h}_{1}(I, \theta)\right)\right| & \leq\left|\frac{\partial^{l}}{\partial I^{l}} h_{0}^{\prime}(I)\right|+\left|\frac{\partial^{l}}{\partial I^{l}} \widetilde{h}_{1}(I, \theta)\right| \\
& \leq C I^{-l+1-2 b}+C I^{-l+1-\gamma-(2 / 5) b} \\
& \leq C I^{-l+1-2 b} .
\end{aligned}
$$

So

$$
\begin{aligned}
& \left|\frac{\partial^{i}}{\partial I^{i}}\left(\frac{1}{h_{0}^{\prime}(I)+\widetilde{h}_{1}(I, \theta)}\right)\right| \\
& \leq C \sum_{l_{1}+\cdots+l_{s}=i}\left|\frac{(-1)^{s} s !}{\left(h_{0}^{\prime}(I)+\widetilde{h}_{1}(I, \theta)\right)^{s+1}}\right| \\
& \times\left|\frac{\partial^{l_{1}}}{\partial I^{l_{1}}}\left(h_{0}^{\prime}(I)+\widetilde{h}_{1}(I, \theta)\right)\right| \\
& \cdots\left|\frac{\partial^{l_{s}}}{\partial I^{l_{s}}}\left(h_{0}^{\prime}(I)+\widetilde{h}_{1}(I, \theta)\right)\right| \\
& \leq C \sum_{l_{1}+\cdots+l_{s}=i} I^{(2 b-1)(s+1)} \cdot I^{-i+(1-2 b) s} \leq C I^{-i+2 b-1} .
\end{aligned}
$$

From (72) and (74), it is easy to see that

$$
\begin{aligned}
& \left|\frac{\partial^{k}}{\partial I^{k}} \eta_{1}(I, \theta)\right| \\
& =\left|\frac{\partial^{k}}{\partial I^{k}}\left(\frac{\widetilde{h}_{1}(I, \theta)}{h_{0}^{\prime}(I)\left(h_{0}^{\prime}(I)+\widetilde{h}_{1}(I, \theta)\right)}\right)\right| \\
& \leq C \sum_{i_{1}+i_{2}+i_{3}=k}\left|\frac{\partial^{i_{1}}}{\partial I^{i_{1}}} \widetilde{h}_{1}(I, \theta)\right| \\
& \leq C \sum_{i_{1}+i_{2}+i_{3}=k} I^{-i_{1}+1-\gamma-(2 / 5) b} I^{-i_{2}-1+2 b} I^{-i_{3}-1+2 b} \\
& \leq C I^{-k-1-\gamma+(3 / 2) b} .
\end{aligned}
$$

(3) We have

$$
\begin{gathered}
\left|\frac{\partial^{k+m}}{\partial I^{k} \partial t^{m}} \widetilde{h}_{2}(I, \theta, t)\right| \leq C I^{-k+\tau}, \\
\left|\frac{\partial^{k+m}}{\partial I^{k} \partial t^{m}} \widetilde{h}_{3}(I, \theta, t)\right| \leq C I^{-k+2-4 b} .
\end{gathered}
$$

By (72), $1-2 b>\tau(\tau=\max (3-6 b,-b))$ and $1-2 b>2-4 b$, we have

$$
\begin{aligned}
\mid h_{0}^{\prime}(I) & +\widetilde{h}_{1}(I, \theta)+\widetilde{h}_{2}(I, \theta, t)+\varepsilon \widetilde{h}_{3}(I, \theta, t) \mid \\
& \geq|| h_{0}^{\prime}(I)+\widetilde{h}_{1}(I, \theta)|-| \widetilde{h}_{2}(I, \theta, t)+\varepsilon \widetilde{h}_{3}(I, \theta, t) \| \\
& \geq\left|h_{0}^{\prime}(I)+\widetilde{h}_{1}(I, \theta)\right|-\left|\widetilde{h}_{2}(I, \theta, t)\right|-\varepsilon\left|\widetilde{h}_{3}(I, \theta, t)\right| \\
& \geq c I^{1-2 b}-C I^{\tau}-C \varepsilon I^{2-4 b} \geq c I^{1-2 b},
\end{aligned}
$$

for $I \gg 1$.

$$
\text { Let } h_{0}^{\prime}(I)+\widetilde{h}_{1}(I, \theta)+\widetilde{h}_{2}(I, \theta, t)+\varepsilon \widetilde{h}_{3}(I, \theta, t)=H(I, \theta, t) \text {. }
$$
We find that

$$
\begin{gathered}
\left|\frac{\partial^{l}}{\partial t^{l}}\left(\frac{1}{\left(h_{0}^{\prime}(I)+\widetilde{h}_{1}(I, \theta)+\widetilde{h}_{2}(I, \theta, t)+\varepsilon \widetilde{h}_{3}(I, \theta, t)\right)^{s+1}}\right)\right| \\
\leq C \sum_{i_{1}+\cdots+i_{r}=l}\left|\frac{(-1)^{r} r !}{(H(I, \theta, t))^{s+1+r}}\right|\left|\frac{\partial^{i_{1}}}{\partial t^{i_{1}}}(H(I, \theta, t))\right| \\
\cdots\left|\frac{\partial^{i_{r}}}{\partial t^{i_{r}}}(H(I, \theta, t))\right| \\
\leq C \sum_{i_{1}+\cdots+i_{r}=l} I^{(2 b-1)(s+1+r)} \cdot \varepsilon^{r} I^{(2-4 b) r} \leq C I^{(2 b-1)(s+1)},
\end{gathered}
$$

so

$$
\begin{gathered}
\left|\frac{\partial^{k+l}}{\partial I^{k} \partial t^{l}}\left(\frac{1}{h_{0}^{\prime}(I)+\widetilde{h}_{1}(I, \theta)+\widetilde{h}_{2}(I, \theta, t)+\varepsilon \widetilde{h}_{3}(I, \theta, t)}\right)\right| \\
\leq C \mid \frac{\partial^{l}}{\partial t^{l}}\left(\sum_{i_{1}+\cdots+i_{s}=k} \frac{(-1)^{s} s !}{(H(I, \theta, t))^{s+1}} \cdot \frac{\partial^{i_{1}}}{\partial I^{i_{1}}}(H(I, \theta, t))\right. \\
\left.\cdots \frac{\partial^{i_{s}}}{\partial I^{i_{s}}}(H(I, \theta, t))\right) \mid \\
\leq C \sum_{i_{1}+\cdots+i_{s}=k} \sum_{j_{0}+j_{1}+\cdots+j_{s}=l}\left|\frac{\partial^{j_{0}}}{\partial t^{j_{0}}} \frac{(-1)^{s} s !}{(H(I, \theta, t))^{s+1}}\right| \\
\times\left|\frac{\partial^{i_{1}+j_{1}}}{\partial I^{i_{1}} \partial t^{j_{1}}}(H(I, \theta, t))\right| \\
\cdots\left|\frac{\partial^{i_{s}+j_{s}}}{\partial I^{i_{s}} \partial t^{j_{s}}}(H(I, \theta, t))\right| \\
\leq C \sum_{i_{1}+\cdots+i_{s}=k} I^{(2 b-1)(s+1)} \cdot I^{-\left(i_{1}+\cdots+i_{s}\right)-(1-2 b) s} \leq C I^{-k+(2 b-1)} .
\end{gathered}
$$

When $m=0$, the proof of (3) is similar to the proof of (2). 
When $m>0$, then

$$
\begin{aligned}
& \left|\frac{\partial^{k+m}}{\partial I^{k} \partial t^{m}} \eta_{2}(I, \theta, t)\right| \\
& =\left|\frac{\partial^{k+m}}{\partial I^{k} \partial t^{m}}\left(\frac{\tilde{h}_{2}(I, \theta, t)}{\left(h_{0}^{\prime}(I)+\widetilde{h}_{1}(I, \theta)\right)\left(h_{0}^{\prime}(I)+\widetilde{h}_{1}+\widetilde{h}_{2}+\varepsilon \widetilde{h}_{3}\right)}\right)\right| \\
& \leq C \mid \frac{\partial^{m}}{\partial t^{m}}\left(\sum_{i_{1}+i_{2}+i_{3}=k} \frac{\partial^{i_{1}}}{\partial I^{i_{1}}} \widetilde{h}_{2} \cdot \frac{\partial^{i_{2}}}{\partial I^{i_{2}}}\left(\frac{1}{h_{0}^{\prime}(I)+\widetilde{h}_{1}}\right)\right. \\
& \left.\cdot \frac{\partial^{i_{3}}}{\partial I^{i_{3}}}\left(\frac{1}{h_{0}^{\prime}(I)+\widetilde{h}_{1}+\widetilde{h}_{2}+\varepsilon \widetilde{h}_{3}}\right)\right) \\
& \leq C \sum_{i_{1}+i_{2}+i_{3}=k} \sum_{l_{1}+l_{2}=m}\left|\frac{\partial^{i_{1}+l_{1}} \tilde{h}_{2}(I, \theta, t)}{\partial I^{i_{1}} \partial t^{l_{1}}}\right| \\
& \cdot\left|\frac{\partial^{i_{2}}}{\partial I^{i_{2}}}\left(\frac{1}{h_{0}^{\prime}(I)+\widetilde{h}_{1}(I, \theta)}\right)\right| \\
& \cdot\left|\frac{\partial^{i_{3}+l_{2}}}{\partial I^{i_{3}} \partial t^{l_{2}}}\left(\frac{1}{h_{0}^{\prime}(I)+\widetilde{h}_{1}+\widetilde{h}_{2}+\varepsilon \widetilde{h}_{3}}\right)\right| \\
& \leq C \sum_{i_{1}+i_{2}+i_{3}=k} I^{-i_{1}+\tau} I^{-i_{2}+2 b-1} I^{-i_{3}+2 b-1} \leq C I^{-k+\tau+4 b-2} .
\end{aligned}
$$

(4) The proof of (4) is similar to the proof of (3).

(5) Let $\eta_{0}(I)+\eta_{1}(I, \theta)+\eta_{2}(I, \theta, t)+\varepsilon \eta_{3}(I, \theta, t)=\eta(I, \theta, t)$. By using the estimates on the functions $\widetilde{l}_{i}(i=1,2)$ and $\eta_{j}(j=0,1,2,3)$, it follows that

$$
\begin{aligned}
& \left|\frac{\partial^{k+m}}{\partial I^{k} \partial t^{m}} \xi_{1}(I, \theta, t)\right| \\
& \leq C\left|\frac{\partial^{m}}{\partial t^{m}}\left(\sum_{k_{1}+k_{2}=k} \frac{\partial^{k_{1}}\left(\widetilde{l}_{1}(I, \theta)+\widetilde{l}_{2}(I, \theta, t)\right)}{\partial I^{k_{1}}} \cdot \frac{\partial^{k_{2}}(\eta(I, \theta, t))}{\partial I^{k_{2}}}\right)\right| \\
& \leq C \sum_{k_{1}+k_{2}=k}\left|\frac{\partial^{k_{1}} \widetilde{l}_{1}(I, \theta)}{\partial I^{k_{1}}} \cdot \frac{\partial^{k_{2}+m}\left(\eta_{2}(I, \theta, t)+\varepsilon \eta_{3}(I, \theta, t)\right)}{\partial I^{k_{2}} \partial t^{m}}\right| \\
& \quad+C \sum_{l_{k}+k_{2}=k} \sum_{m_{1}+m_{2}=m}\left|\frac{\partial^{k_{1}+m_{1}} \tilde{l}_{2}(I, \theta, t)}{\partial I^{k_{1}} \partial t^{m_{1}}} \cdot \frac{\partial^{k_{2}+m_{2}}(\eta(I, \theta, t))}{\partial I^{k_{2}} \partial t^{m_{2}}}\right| \\
& \leq C I^{-k_{1}+2-\gamma-(5 / 2) b} \cdot\left(I^{-k_{2}+\tau+4 b-2}+\varepsilon I^{-k_{2}}\right)+C I^{-k_{1}+a} \cdot I^{-k_{2}+2 b-1} \\
& \leq C I^{-k+a+2 b-1},
\end{aligned}
$$

when $m \neq 0$.

When $m=0$, then

$$
\begin{aligned}
& \left|\frac{\partial^{k}}{\partial I^{k}} \xi_{1}(I, \theta, t)\right| \\
& \quad \leq C \sum_{k_{1}+k_{2}=k}\left|\frac{\partial^{k_{1}}\left(\widetilde{l}_{1}(I, \theta)+\widetilde{l}_{2}(I, \theta, t)\right)}{\partial I^{k_{1}}} \cdot \frac{\partial^{k_{2}}(\eta(I, \theta, t))}{\partial I^{k_{2}}}\right| \\
& \quad \leq C\left(I^{-k_{1}+2-\gamma-(5 / 2) b}+I^{-k_{1}+a}\right) \cdot I^{-k_{2}+2 b-1} \leq C I^{-k+a+2 b-1}
\end{aligned}
$$

by $a>2-\gamma-5 b / 2$.
(6) By using the estimates on the functions $\widetilde{l}_{3}$ and $\eta_{i}(i=$ $0,1,2,3)$, it follows that

$$
\begin{aligned}
& \left|\frac{\partial^{k+m}}{\partial I^{k} \partial t^{m}} \xi_{2}(I, \theta, t)\right| \\
& \quad \leq C\left|\frac{\partial^{m}}{\partial t^{m}}\left(\sum_{k_{1}+k_{2}=k} \frac{\partial^{k_{1}}\left(\tilde{l}_{3}(I, \theta, t)\right)}{\partial I^{k_{1}}} \cdot \frac{\partial^{k_{2}}(\eta(I, \theta, t))}{\partial I^{k_{2}}}\right)\right| \\
& \quad \leq C \sum_{l_{k}+k_{2}=k} \sum_{m_{1}+m_{2}=m}\left|\frac{\partial^{k_{1}+m_{1}} \tilde{l}_{3}(I, \theta, t)}{\partial I^{k_{1}} \partial t^{m_{1}}} \cdot \frac{\partial^{k_{2}+m_{2}}(\eta(I, \theta, t))}{\partial I^{k_{2}} \partial t^{m_{2}}}\right| \\
& \quad \leq C I^{-k_{1}+3-4 b} \cdot I^{-k_{2}+2 b-1} \\
& \quad \leq C I^{-k+2-2 b} .
\end{aligned}
$$

(7) By using the estimates on the functions $\widetilde{l}_{4}$ and $\eta_{i}(i=$ $0,1,2,3)$, it follows that

$$
\begin{aligned}
& \left|\frac{\partial^{k+m}}{\partial I^{k} \partial t^{m}} \xi_{3}(I, \theta, t)\right| \\
& \leq C\left|\frac{\partial^{m}}{\partial t^{m}}\left(\sum_{k_{1}+k_{2}=k} \frac{\partial^{k_{1}}\left(\widetilde{l}_{4}(I, \theta, t)\right)}{\partial I^{k_{1}}} \cdot \frac{\partial^{k_{2}}(\eta(I, \theta, t))}{\partial I^{k_{2}}}\right)\right| \\
& \quad \leq C \sum_{l_{k}+k_{2}=k m_{1}+m_{2}=m}\left|\frac{\partial^{k_{1}+m_{1}} \widetilde{l}_{4}(I, \theta, t)}{\partial I^{k_{1}} \partial t^{m_{1}}} \cdot \frac{\partial^{k_{2}+m_{2}}(\eta(I, \theta, t))}{\partial I^{k_{2}} \partial t^{m_{2}}}\right| \\
& \leq C I^{-k_{1}+3-4 b} \cdot I^{-k_{2}+2 b-1} \\
& \leq C I^{-k+2-2 b} \cdot \\
& \text { Let } t=t, \theta=\theta, r=\eta_{0}(I) \text { and } \\
& F_{0}(r, \theta)=\eta_{1}(I(r), \theta) \\
& F_{1}(r, \theta, t)=\eta_{2}(I(r), \theta, t)+\varepsilon \eta_{3}(I(r), \theta, t) \\
& F_{3}(r, \theta, t)=\varepsilon \eta_{0}^{\prime}(I(r)) \cdot \xi_{3}(I(r), \theta, t) \\
& F_{2}(r, \theta, t)=\eta_{0}^{\prime}(I(r)) \cdot\left(\xi_{1}(I(r), \theta, t)+\varepsilon \xi_{2}(I(r), \theta, t)\right)
\end{aligned}
$$

where $I(r)$ is the inverse function of $r=\eta_{0}(I)$.

Then system (70) is transformed into the following form:

$$
\begin{gathered}
\frac{d t}{d \theta}=r+F_{0}(r, \theta)+F_{1}(r, \theta, t) \\
\frac{d r}{d \theta}=F_{2}(r, \theta, t)+\alpha T_{0}\left|S\left(\theta T_{0}\right)\right|^{\alpha-1} C\left(\theta T_{0}\right) \cdot F_{3}(r, \theta, t) .
\end{gathered}
$$

Moreover, one can verify that system (86) is reversible with respect to involution $G:(t, r) \mapsto(-t, r)$. 
It is easy to see that $I \gg 1$ if and only if $r \gg 1$, and the solutions of system (86) do exist on $0 \leq \theta \leq 1$ when $r(0)=$ $r \gg 1$.

By using the estimates on $\eta_{i}$ and $\xi_{i}(i=1,2,3)$ in Lemma 10, the following inequalities can be proved.

Lemma 11. For $0 \leq k+m \leq 4$ and $r \gg 1$, the following inequalities hold:

(1) $\left|\left(\partial^{k} / \partial r^{k}\right) F_{0}(r, \theta)\right| \leq C r^{-k-(1+\gamma-3 b / 2) /(2 b-1)}$,

(2) $\left|\left(\partial^{k+m} / \partial r^{k} \partial t^{m}\right) F_{1}(r, \theta, t)\right| \leq C\left(r^{-k+(\tau+4 b-2) /(2 b-1)}+\right.$ $\left.\varepsilon r^{-k}\right)$

(3) $\left|\left(\partial^{k+m} / \partial r^{k} \partial t^{m}\right) F_{2}(r, \theta, t)\right| \leq C\left(r^{-k+(a+4 b-3) /(2 b-1)}+\right.$ $\left.\varepsilon r^{-k}\right)$,

(4) $\left|\left(\partial^{k+m} / \partial r^{k} \partial t^{m}\right) F_{3}(r, \theta, t)\right| \leq C \varepsilon r^{-k}$.

Proof. Above all, we know that $r=\eta_{0}(I)=T_{0} I^{2 b-1}$, so we can get $I=\left(\left(1 / T_{0}\right) r\right)^{1 /(2 b-1)}$. Then we have

$$
\begin{gathered}
\left|\frac{d^{j} I}{d r^{j}}\right| \leq C r^{-j+1 /(2 b-1)}, \\
\left|\frac{d^{j} \eta_{0}^{\prime}(I(r))}{d r^{j}}\right| \leq C\left|\frac{d^{j}\left(r^{1-1 /(2 b-1)}\right)}{d r^{j}}\right| \leq C r^{-j+(2 b-2) /(2 b-1)} .
\end{gathered}
$$

(1) We have that

$$
\begin{aligned}
& \left|\frac{\partial^{k} F_{0}(r, \theta)}{\partial r^{k}}\right| \\
& \quad \leq \sum_{k_{1}+\cdots+k_{s}=k}\left|\frac{\partial^{s} \eta_{1}(I, \theta)}{\partial I^{s}}\right| \cdot\left|\frac{d^{k_{1}} I}{d r^{k_{1}}}\right| \cdots\left|\frac{d^{k_{s}} I}{d r^{k_{s}}}\right| \\
& \quad \leq C I^{-s-1-\gamma+(3 / 2) b} r^{-k+(1 /(2 b-1)) s} \\
& \quad \leq C r^{-s(1 /(2 b-1))-(1+\gamma-(3 / 2) b) /(2 b-1)} r^{-k+(1 /(2 b-1)) s} \\
& \quad \leq C r^{-k-(1+\gamma-(3 / 2) b) /(2 b-1)} .
\end{aligned}
$$

(2) We have that

$$
\begin{aligned}
& \left|\frac{\partial^{k+m} F_{1}(r, \theta, t)}{\partial r^{k} \partial t^{m}}\right| \\
& \leq C \sum_{i_{1}+\cdots+i_{s}=k}\left|\frac{\partial^{s+m} \eta_{2}(I, \theta, t)}{\partial I^{s} \partial t^{m}} \frac{d^{i_{1}} I}{d r^{i_{1}}} \cdots \frac{d^{i_{s}} I}{d r^{i_{s}}}\right| \\
& \quad+C \varepsilon \sum_{j_{1}+\cdots+j_{\nu}=k}\left|\frac{\partial^{\nu+m} \eta_{3}(I, \theta, t)}{\partial I^{v} \partial t^{m}} \frac{d^{j_{1}} I}{d r^{j_{1}}} \cdots \frac{d^{j_{\nu}} I}{d r^{j_{v}}}\right| \\
& \leq C\left(r^{-k+((\tau+4 b-2) /(2 b-1))}+\varepsilon r^{-k}\right) .
\end{aligned}
$$

(3) We have that

$$
\begin{aligned}
& \left|\frac{\partial^{k+m} F_{2}(r, \theta, t)}{\partial r^{k} \partial t^{m}}\right| \\
& \leq \mid \sum_{k_{1}+k_{2}=k} \frac{d^{k_{1}} \eta_{0}^{\prime}(I(r))}{d r^{k_{1}}} \\
& \cdot\left(\frac{\partial^{k_{2}+m} \xi_{1}(I(r), \theta, t)}{\partial r^{k_{2}} \partial t^{m}}+\varepsilon \frac{\partial^{k_{2}+m} \xi_{2}(I(r), \theta, t)}{\partial r^{k_{2}} \partial t^{m}}\right) \mid \\
& \leq C \sum_{k_{1}+k_{2}=k} r^{-k_{1}+(2 b-2) /(2 b-1)} \\
& \times\left(\sum_{i_{1}+\cdots+i_{s}=k_{2}} \frac{\partial^{s+m} \xi_{1}(I, \theta, t)}{\partial I^{s} \partial t^{m}} \frac{d^{i_{1}} I}{d r^{i_{1}}} \cdots \frac{d^{i_{s}} I}{d r^{i_{s}}}\right. \\
& \left.+\varepsilon \sum_{j_{1}+\cdots+j_{v}=k_{2}} \frac{\partial^{\nu+m} \xi_{2}(I, \theta, t)}{\partial I^{v} \partial t^{m}} \frac{d^{j_{1}} I}{d r^{j_{1}}} \cdots \frac{d^{j_{\nu}} I}{d r^{j_{\nu}}}\right) \\
& \leq C r^{-k_{1}+(2 b-2) /(2 b-1)} r^{(1 /(2 b-1))(-s+a+2 b-1)} r^{-k_{1}+(1 /(2 b-1)) s} \\
& +C \varepsilon r^{-k_{1}+(2 b-2) /(2 b-1)} r^{(1 /(2 b-1))(-\gamma+2-2 b)} r^{-k_{1}+(1 /(2 b-1)) v} \\
& \leq C r^{-k+(a+4 b-3) /(2 b-1)}+C \varepsilon r^{-k} \text {. }
\end{aligned}
$$

(4) We have that

$$
\begin{aligned}
& \left|\frac{\partial^{k+m} F_{3}(r, \theta, t)}{\partial r^{k} \partial t^{m}}\right| \\
& \quad \leq \varepsilon\left|\sum_{k_{1}+k_{2}=k} \frac{d^{k_{1}} \eta_{0}^{\prime}(I(r))}{d r^{k_{1}}} \cdot\left(\frac{\partial^{k_{2}+m} \xi_{3}(I(r), \theta, t)}{\partial r^{k_{2}} \partial t^{m}}\right)\right| \\
& \quad \leq C \varepsilon \sum_{k_{1}+k_{2}=k} r^{-k_{1}+(2 b-2) /(2 b-1)} \\
& \quad \times\left(\sum_{i_{1}+\cdots+i_{s}=k_{2}} \frac{\partial^{s+m} \xi_{3}(I, \theta, t)}{\partial I^{s} \partial t^{m}} \frac{d^{i_{1}} I}{d r^{i_{1}}} \cdots \frac{d^{i_{s}} I}{d r^{i_{s}}}\right) \\
& \leq C \varepsilon r^{-k} .
\end{aligned}
$$

Lemma 12. The time 1 map $\Phi^{1}$ of the flow $\Phi^{\theta}$ of the system (86) is of the form

$$
\Phi^{1}: r_{1}=r+Q_{2}(r, t), \quad t_{1}=t+\widehat{\omega}(r)+Q_{1}(r, t),
$$

where $\widehat{\omega}(r)=r+\int_{0}^{1} F_{0}(r, \theta) d \theta$. And there exists a $\mu_{0}>0$ such that, for $0 \leq k+m \leq 4$, sufficiently large $r$ and sufficiently small $\varepsilon$,

$$
\left|\frac{\partial^{k+m}}{\partial r^{k} \partial t^{m}} Q_{i}(r, t)\right| \leq C r^{-\mu_{0}}+\varepsilon, \quad i=1,2
$$


hold. Moreover, the map $\Phi^{1}$ is reversible with respect to the involution $G:(t, r) \mapsto(-t, r)$.

Proof. Since

$$
\begin{aligned}
& \int_{0}^{1} \alpha T_{0}\left|S\left(\theta T_{0}\right)\right|^{\alpha-1}\left|C\left(\theta T_{0}\right)\right| d \theta \\
& =\lim _{\epsilon \rightarrow 0^{+}} \int_{\epsilon}^{1 / 4} \alpha T_{0}\left|S\left(\theta T_{0}\right)\right|^{\alpha-1} C\left(\theta T_{0}\right) d \theta \\
& -\lim _{\epsilon \rightarrow 0^{+}} \int_{1 / 4}^{1 / 2-\epsilon} \alpha T_{0}\left|S\left(\theta T_{0}\right)\right|^{\alpha-1} C\left(\theta T_{0}\right) d \theta \\
& -\lim _{\epsilon \rightarrow 0^{+}} \int_{1 / 2+\epsilon}^{3 / 4} \alpha T_{0}\left|S\left(\theta T_{0}\right)\right|^{\alpha-1} C\left(\theta T_{0}\right) d \theta \\
& +\lim _{\epsilon \rightarrow 0^{+}} \int_{3 / 4}^{1-\epsilon} \alpha T_{0}\left|S\left(\theta T_{0}\right)\right|^{\alpha-1} C\left(\theta T_{0}\right) d \theta \\
& =\left|S\left(\frac{T_{0}}{4}\right)\right|^{\alpha-1} S\left(\frac{T_{0}}{4}\right)-\lim _{\epsilon \rightarrow 0^{+}}\left|S\left(\epsilon T_{0}\right)\right|^{\alpha-1} S\left(\epsilon T_{0}\right) \\
& -\left[\lim _{\epsilon \rightarrow 0^{+}}\left|S\left(\left(\frac{1}{2}-\epsilon\right) T_{0}\right)\right|^{\alpha-1} S\left(\left(\frac{1}{2}-\epsilon\right) T_{0}\right)\right. \\
& \left.-\left|S\left(\frac{T_{0}}{4}\right)\right|^{\alpha-1} S\left(\frac{T_{0}}{4}\right)\right] \\
& -\left[\left|S\left(\frac{3 T_{0}}{4}\right)\right|^{\alpha-1} S\left(\frac{3 T_{0}}{4}\right)\right. \\
& \left.-\lim _{\epsilon \rightarrow 0^{+}}\left|S\left(\left(\frac{1}{2}+\epsilon\right) T_{0}\right)\right|^{\alpha-1} S\left(\left(\frac{1}{2}+\epsilon\right) T_{0}\right)\right] \\
& +\left[\left.\lim _{\epsilon \rightarrow 0^{+}} S\left((1-\epsilon) T_{0}\right)\right|^{\alpha-1} S\left((1-\epsilon) T_{0}\right)\right. \\
& \left.-\left|S\left(\frac{3 T_{0}}{4}\right)\right|^{\alpha-1} S\left(\frac{3 T_{0}}{4}\right)\right] \\
& =4\left|S\left(\frac{T_{0}}{4}\right)\right|^{\alpha-1} S\left(\frac{T_{0}}{4}\right)=4 \text {, }
\end{aligned}
$$

then we get $\int_{0}^{1} \alpha T_{0}\left|S\left(\theta T_{0}\right)\right|^{\alpha-1}\left|C\left(\theta T_{0}\right)\right| d \theta$ is bounded.

Let $\alpha T_{0}\left|S\left(v T_{0}\right)\right|^{\alpha-1} C\left(v T_{0}\right)=S_{1}(v)$. Set $(r(\theta), t(\theta))=$ $\Phi^{\theta}(r, t)$ with $\Phi^{0}=i d$ for the flow:

$$
t(\theta)=t+r \theta+D_{1}(r, t, \theta), \quad r(\theta)=r+D_{2}(r, t, \theta) .
$$

Since

$$
\Phi^{\theta}=\Phi^{0}+\int_{0}^{\theta} X \cdot \Phi^{v} d v
$$

where $X$ denotes the vector field of the system (86), we have

$$
\begin{aligned}
t(\theta)= & t \\
& +\int_{0}^{\theta}\left[r(v)+F_{0}(r(v), v)+F_{1}(r(v), v, t(v))\right] d v \\
= & t+r \theta \\
& +\int_{0}^{\theta}\left[D_{2}(r, t, v)+F_{0}\left(r+D_{2}, v\right)\right. \\
& \left.\quad+F_{1}\left(r+D_{2}, v, t+r v+D_{1}\right)\right] d v \\
= & t+r \theta+D_{1}(r, t, \theta), \\
r(\theta)= & r \quad+\int_{0}^{\theta}\left[F_{2}(r(v), v, t(v))\right. \\
& \left.\quad+S_{1}(v) F_{3}(r(v), v, t(v))\right] d v \\
& +\int_{0}^{\theta}\left[F_{2}\left(r+D_{2}, v, t+r v+D_{1}\right)\right. \\
\quad & \left.+S_{1}(v) F_{3}\left(r+D_{2}, v, t+r v+D_{1}\right)\right] d v \\
= & r+D_{2}(r, t, \theta),
\end{aligned}
$$

which is equivalent to the following equations for $D_{1}$ and $D_{2}$ :

$$
\begin{aligned}
D_{1}(r, t, \theta)=\int_{0}^{\theta}[ & D_{2}(r, t, v)+F_{0}\left(r+D_{2}, v\right) \\
+ & \left.F_{1}\left(r+D_{2}, v, t+r v+D_{1}\right)\right] d v, \\
D_{2}(r, t, \theta)=\int_{0}^{\theta}[ & F_{2}\left(r+D_{2}, v, t+r v+D_{1}\right) \\
& \left.+S_{1}(v) F_{3}\left(r+D_{2}, v, t+r v+D_{1}\right)\right] d v .
\end{aligned}
$$

Let $D(r, t, \theta)=\left(D_{1}(r, t, \theta), D_{2}(r, t, \theta)\right),\left|D_{1}(r, t, \theta)\right|=$ $\sup _{\substack{(r, t) \in) \in \\\left(R^{+} \times \mathbb{T} \times(0,1)\right.}}\left|D_{1}(r, t, \theta)\right|$. Define $\|D\|=:\left|D_{1}\right| / 3+2\left|D_{1}\right| / 3$, and $T(D)=:\left(T_{1}(D), T_{2}(D)\right)$, where

$$
\begin{aligned}
T_{1}(D)=\int_{0}^{\theta}[ & D_{2}(r, t, v)+F_{0}\left(r+D_{2}, v\right) \\
+ & \left.F_{1}\left(r+D_{2}, v, t+r v+D_{1}\right)\right] d v, \\
T_{2}(D)=\int_{0}^{\theta}[ & F_{2}\left(r+D_{2}, v, t+r v+D_{1}\right) \\
+ & \left.S_{1}(v) F_{3}\left(r+D_{2}, v, t+r v+D_{1}\right)\right] d v .
\end{aligned}
$$


Next, we will prove that $T$ is a contraction map. From the definition of $T(D)$, we have

$$
\begin{aligned}
& \left|T_{1} D-T_{1} \widetilde{D}\right| \\
& =\mid \int_{0}^{\theta}\left[D_{2}-\widetilde{D}_{2}+F_{0}\left(r+D_{2}, v\right)-F_{0}\left(r+\widetilde{D}_{2}\right)\right. \\
& +F_{1}\left(r+D_{2}, v, t+r v+D_{1}\right) \\
& \left.-F_{1}\left(r+\widetilde{D}_{2}, v, t+r v+\widetilde{D}_{1}\right)\right] d v \\
& \leq\left|D_{2}-\widetilde{D}_{2}\right| \\
& +\int_{0}^{1}\left|\frac{\partial F_{0}\left(r+s\left(D_{2}-\widetilde{D}_{2}\right), v\right)}{\partial r}\right| \cdot\left|D_{2}-\widetilde{D}_{2}\right| d s \\
& +\int_{0}^{1}\left|\frac{\partial F_{1}\left(r+s\left(D_{2}-\widetilde{D}_{2}\right), v, t+r v+D_{1}\right)}{\partial r}\right| \\
& \cdot\left|D_{2}-\widetilde{D}_{2}\right| d s \\
& +\int_{0}^{1}\left|\frac{\partial F_{1}\left(r+\widetilde{D}_{2}, v, t+r v+s\left(D_{1}-\widetilde{D}_{1}\right)\right)}{\partial t}\right| \\
& \cdot\left|D_{1}-\widetilde{D}_{1}\right| d s \\
& \leq \frac{6}{5}\left|D_{2}-\widetilde{D}_{2}\right|+\frac{1}{4}\left|D_{1}-\widetilde{D}_{1}\right| \text {, } \\
& \left|T_{2} D-T_{2} \widetilde{D}\right| \\
& =\int_{0}^{\theta}\left[F_{2}\left(r+D_{2}, v, t+r v+D_{1}\right)\right. \\
& -F_{2}\left(r+\widetilde{D}_{2}, v, t+r v+\widetilde{D}_{1}\right) \\
& +S_{1}(v) F_{3}\left(r+D_{2}, v, t+r v+D_{1}\right) \\
& \left.-S_{1}(v) F_{3}\left(r+\widetilde{D}_{2}, v, t+r v+\widetilde{D}_{1}\right)\right] d v \\
& \leq \int_{0}^{1}\left|\frac{\partial F_{2}\left(r+s\left(D_{2}-\widetilde{D}_{2}\right), v, t+r v+D_{1}\right)}{\partial r}\right| \\
& \cdot\left|D_{2}-\widetilde{D}_{2}\right| d s \\
& +\int_{0}^{1}\left|\frac{\partial F_{2}\left(r+\widetilde{D}_{2}, v, t+r v+s\left(D_{1}-\widetilde{D}_{1}\right)\right)}{\partial t}\right| \\
& \cdot\left|D_{1}-\widetilde{D}_{1}\right| d s \\
& +\int_{0}^{1}\left|S_{1}(v)\right| d v \\
& \cdot \int_{0}^{1}\left|\frac{\partial F_{3}\left(r+s\left(D_{2}-\widetilde{D}_{2}\right), v, t+r v+D_{1}\right)}{\partial r}\right| \\
& \cdot\left|D_{2}-\widetilde{D}_{2}\right| d s
\end{aligned}
$$

$$
\begin{aligned}
& +\int_{0}^{1}\left|S_{1}(v)\right| d v \\
& \cdot \int_{0}^{1}\left|\frac{\partial F_{3}\left(r+\widetilde{D}_{2}, v, t+r v+s\left(D_{1}-\widetilde{D}_{1}\right)\right)}{\partial t}\right| \\
& \cdot\left|D_{1}-\widetilde{D}_{1}\right| d s \\
& \leq \frac{3}{20}\left|D_{2}-\widetilde{D}_{2}\right|+\frac{1}{8}\left|D_{1}-\widetilde{D}_{1}\right|
\end{aligned}
$$

by Lemma 11 and the boundedness of $\int_{0}^{1}\left|S_{1}(v)\right| d v$. Then we have

$$
\begin{aligned}
\| T(D) & -T(\widetilde{D}) \| \\
= & \frac{1}{3}\left|T_{1}(D)-T_{1}(\widetilde{D})\right|+\frac{2}{3}\left|T_{2}(D)-T_{2}(\widetilde{D})\right| \\
\leq & \frac{1}{3} \times\left(\frac{6}{5}\left|D_{2}-\widetilde{D}_{2}\right|+\frac{1}{4}\left|D_{1}-\widetilde{D}_{1}\right|\right) \\
& +\frac{2}{3} \times\left(\frac{3}{20}\left|D_{2}-\widetilde{D}_{2}\right|+\frac{1}{8}\left|D_{1}-\widetilde{D}_{1}\right|\right) \\
= & \frac{1}{6}\left|D_{1}-\widetilde{D}_{1}\right|+\frac{1}{2}\left|D_{2}-\widetilde{D}_{2}\right| \\
\leq & \frac{3}{4} \times\left(\frac{1}{3}\left|D_{1}-\widetilde{D}_{1}\right|+\frac{2}{3}\left|D_{2}-\widetilde{D}_{2}\right|\right) \\
\leq & \frac{3}{4}\|D-\widetilde{D}\|,
\end{aligned}
$$

by the definition of the norm $\|\cdot\|$.

Using the contraction principle, one verifies easily that for $r \geq r_{0}$, (98) has a unique solution in the space $\left\{\left|D_{1}\right| \leq\right.$ $\left.1,\left|D_{2}\right| \leq 1\right\}$. Moreover, $D_{1}$ and $D_{2}$ are smooth.

Next, we will estimate $Q_{1}(r, t)$ and $Q_{2}(r, t)$ as follows:

$$
\begin{aligned}
Q_{1}(r, t)= & D_{1}(r, t, 1)-\int_{0}^{1} F_{0}(r, v) d v \\
= & \int_{0}^{1}\left[D_{2}(r, t, v)\right. \\
& +\int_{0}^{1} \frac{\partial F_{0}\left(r+s D_{2}, v\right)}{\partial r} \cdot D_{2} d s \\
& \left.+F_{1}\left(r+D_{2}, v, t+r v+D_{1}\right)\right] d v
\end{aligned}
$$

$Q_{2}(r, t)=D_{2}(r, t, 1)$

$$
\begin{aligned}
=\int_{0}^{1}[ & F_{2}\left(r+D_{2}, v, t+r v+D_{1}\right) \\
& \left.+S_{1}(v) F_{3}\left(r+D_{2}, v, t+r v+D_{1}\right)\right] d v .
\end{aligned}
$$


In order to prove (93), we just need to prove that

$$
\left|\frac{\partial^{k+m}}{\partial r^{k} \partial t^{m}} D_{i}(r, t, \theta)\right| \leq C r^{-\mu_{0}}+C \varepsilon, \quad i=1,2
$$

hold for $k+m \leq 4$.

(1) When $k+m=0$,

$$
\begin{aligned}
& \left|D_{2}(r, t, \theta)\right| \\
& \leq \int_{0}^{\theta}\left(\left|F_{2}\left(r+D_{2}, v, t+r v+D_{1}\right)\right|\right. \\
& \left.+\left|S_{1}(v)\right|\left|F_{3}\left(r+D_{2}, v, t+r v+D_{1}\right)\right|\right) d v \\
& \leq C\left(r^{-\mu_{0}}+\varepsilon\right)+\int_{0}^{1}\left|S_{1}(v)\right| d v \cdot(C \varepsilon) \leq C\left(r^{-\mu_{0}}+\varepsilon\right), \\
& \left|D_{1}(r, t, \theta)\right| \\
& \leq\left|D_{2}(r, t, \theta)\right| \\
& +\int_{0}^{\theta}\left(\left|F_{0}\left(r+D_{2}, v\right)\right|+\left|F_{1}\left(r+D_{2}, v, t+r v+D_{1}\right)\right|\right) d v \text {, } \\
& \leq\left|D_{2}(r, t, v)\right|+\int_{0}^{\theta}\left(C r^{-\mu_{0}}+C r^{-\mu_{0}}+C \varepsilon\right) d v \\
& \leq\left|D_{2}(r, t, \theta)\right|+C\left(r^{-\mu_{0}}+\varepsilon\right) \leq C\left(r^{-\mu_{0}}+\varepsilon\right),
\end{aligned}
$$

where $\mu_{0}=\min ((1+\gamma-3 b / 2) /(2 b-1),(2-4 b-\tau) /(2 b-$ $1),(3-4 b-a) /(2 b-1))$.

(2) When $m=0$ and $k \neq 0$, we check the case when $k=1$ firstly

$$
\begin{aligned}
& \left|\frac{\partial D_{2}(r, t, \theta)}{\partial r}\right| \\
& \leq \int_{0}^{\theta}\left|\frac{\partial F_{2}\left(r+D_{2}, v, t+r v+D_{1}\right)}{\partial r}\right| \\
& \cdot\left(1+\left|\frac{\partial D_{2}(r, t, v)}{\partial r}\right|\right) d v \\
& +\int_{0}^{\theta}\left|\frac{\partial F_{2}\left(r+D_{2}, v, t+r v+D_{1}\right)}{\partial t}\right| \\
& \cdot\left(1+\left|\frac{\partial D_{1}(r, t, v)}{\partial r}\right|\right) d v \\
& +\int_{0}^{1}\left|S_{1}(v)\right| d v \cdot \int_{0}^{\theta}\left|\frac{\partial F_{3}\left(r+D_{2}, v, t+r v+D_{1}\right)}{\partial r}\right| \\
& \cdot\left(1+\left|\frac{\partial D_{2}(r, t, v)}{\partial r}\right|\right) d v \\
& +\int_{0}^{1}\left|S_{1}(v)\right| d v \cdot \int_{0}^{\theta}\left|\frac{\partial F_{3}\left(r+D_{2}, v, t+r v+D_{1}\right)}{\partial t}\right| \\
& \cdot\left(1+\left|\frac{\partial D_{1}(r, t, v)}{\partial r}\right|\right) d v \\
& \leq C r^{-1}\left(r^{-\mu_{0}}+\varepsilon\right) \cdot\left(1+\left|\frac{\partial D_{2}(r, t, v)}{\partial r}\right|\right) \\
& +C\left(r^{-\mu_{0}}+\varepsilon\right) \cdot\left(1+\left|\frac{\partial D_{1}(r, t, v)}{\partial r}\right|\right),
\end{aligned}
$$

$$
\begin{aligned}
& \left|\frac{\partial D_{1}(r, t, \theta)}{\partial r}\right| \\
& \leq\left|\frac{\partial D_{2}(r, t, \theta)}{\partial r}\right| \\
& +\int_{0}^{\theta}\left|\frac{\partial F_{0}\left(r+D_{2}, v\right)}{\partial r}\right| \cdot\left(1+\left|\frac{\partial D_{2}(r, t, v)}{\partial r}\right|\right) d v \\
& +\int_{0}^{\theta}\left|\frac{\partial F_{1}\left(r+D_{2}, v, t+r v+D_{1}\right)}{\partial r}\right| \cdot\left(1+\left|\frac{\partial D_{2}(r, t, v)}{\partial r}\right|\right) d v \\
& +\int_{0}^{\theta}\left|\frac{\partial F_{1}\left(r+D_{2}, v, t+r v+D_{1}\right)}{\partial t}\right| \cdot\left(1+\left|\frac{\partial D_{1}(r, t, v)}{\partial r}\right|\right) d v \\
& \leq\left|\frac{\partial D_{2}(r, t, \theta)}{\partial r}\right|+C r^{-1}\left(r^{-\mu_{0}}+\varepsilon\right) \cdot\left(1+\left|\frac{\partial D_{2}(r, t, v)}{\partial r}\right|\right) \\
& +C\left(r^{-\mu_{0}}+\varepsilon\right) \cdot\left(1+\left|\frac{\partial D_{1}(r, t, v)}{\partial r}\right|\right) \text {. }
\end{aligned}
$$

Hence,

$$
\begin{aligned}
& \left|\frac{\partial D_{1}(r, t, \theta)}{\partial r}\right| \leq C\left(r^{-\mu_{0}}+\varepsilon\right), \\
& \left|\frac{\partial D_{2}(r, t, \theta)}{\partial r}\right| \leq C\left(r^{-\mu_{0}}+\varepsilon\right) .
\end{aligned}
$$

Now, we proceed inductively by assuming that for $j<k-1$ the estimates

$$
\begin{aligned}
& \left|\frac{\partial^{j} D_{1}(r, t, \theta)}{\partial r^{j}}\right| \leq C\left(r^{-\mu_{0}}+\varepsilon\right), \\
& \left|\frac{\partial^{j} D_{2}(r, t, \theta)}{\partial r^{j}}\right| \leq C\left(r^{-\mu_{0}}+\varepsilon\right),
\end{aligned}
$$

hold and we wish to conclude that the same estimate holds for $j=k$

$$
\begin{aligned}
& \left|\frac{\partial^{k} D_{2}(r, t, \theta)}{\partial r^{k}}\right| \\
& \leq \int_{0}^{\theta}\left|\frac{\partial F_{2}\left(r+D_{2}, v, t+r v+D_{1}\right)}{\partial r}\right| \\
& \quad \cdot\left|\frac{\partial^{k} D_{2}(r, t, \theta)}{\partial r^{k}}\right| d v \\
& +\int_{0}^{\theta}\left|\frac{\partial F_{2}\left(r+D_{2}, v, t+r v+D_{1}\right)}{\partial t}\right| \\
& +C\left(r^{\left.-\mu_{0}+\varepsilon\right)} \mid d v\right. \\
& \quad \sum_{k_{1}+k_{2}=k_{j_{1}+\ldots+i_{s}=k_{1}}}\left|\frac{\partial^{k} D_{1}(r, t, v)}{\partial r^{k}}\right| \frac{\partial^{i_{1}}\left(r+D_{2}\right)}{\partial r^{i_{1}}}|\ldots| \frac{\partial^{i_{s}}\left(r+D_{2}\right)}{\partial r^{i_{s}}} \mid \\
&
\end{aligned}
$$




$$
\begin{aligned}
& +\int_{0}^{1}\left|S_{1}(v)\right| d v \\
& \cdot \int_{0}^{\theta}\left|\frac{\partial F_{3}\left(r+D_{2}, v, t+r v+D_{1}\right)}{\partial r}\right| \cdot\left|\frac{\partial^{k} D_{2}(r, t, v)}{\partial r^{k}}\right| d v \\
& +\int_{0}^{1}\left|S_{1}(v)\right| d v \\
& \cdot \int_{0}^{\theta}\left|\frac{\partial F_{3}\left(r+D_{2}, v, t+r v+D_{1}\right)}{\partial t}\right| \cdot\left|\frac{\partial^{k} D_{1}(r, t, v)}{\partial r^{k}}\right| d v \\
& +C\left(r^{-\mu_{0}}+\varepsilon\right) \\
& \cdot \sum_{k_{1}+k_{2}=k} \sum_{\substack{i_{1}+\cdots+i_{s}=k_{1} \\
j_{1}+\cdots+j_{v}=k_{2}}}\left|\frac{\partial^{i_{1}}\left(r+D_{2}\right)}{\partial r^{i_{1}}}\right| \cdots\left|\frac{\partial^{i_{s}}\left(r+D_{2}\right)}{\partial r^{i_{s}}}\right| \\
& \times\left|\frac{\partial^{j_{1}}\left(r+D_{1}\right)}{\partial r^{j_{1}}}\right| \cdots\left|\frac{\partial^{j_{v}}\left(r+D_{1}\right)}{\partial r^{j_{v}}}\right| \\
& \leq C r^{-1}\left(r^{-\mu_{0}}+\varepsilon\right) \cdot\left|\frac{\partial^{k} D_{2}(r, t, v)}{\partial r^{k}}\right| \\
& +C\left(r^{-\mu_{0}}+\varepsilon\right) \cdot\left|\frac{\partial^{k} D_{1}(r, t, v)}{\partial r^{k}}\right|+C\left(r^{-\mu_{0}}+\varepsilon\right), \\
& \left|\frac{\partial^{k} D_{1}(r, t, \theta)}{\partial r^{k}}\right| \\
& \leq\left|\frac{\partial^{k} D_{2}(r, t, \theta)}{\partial r^{k}}\right| \\
& +\int_{0}^{\theta}\left|\frac{\partial F_{0}\left(r+D_{2}, v\right)}{\partial r}\right| \cdot\left|\frac{\partial^{k} D_{2}(r, t, v)}{\partial r^{k}}\right| d v \\
& +C\left(r^{-\mu_{0}}+\varepsilon\right) \cdot \sum_{i_{1}+\cdots+i_{s}=k}\left|\frac{\partial^{i_{1}}\left(r+D_{2}\right)}{\partial r^{i_{1}}}\right| \cdots\left|\frac{\partial^{i_{s}}\left(r+D_{2}\right)}{\partial r^{i_{s}}}\right| \\
& +\int_{0}^{\theta}\left|\frac{\partial F_{1}\left(r+D_{2}, v, t+r v+D_{1}\right)}{\partial r}\right| \cdot\left|\frac{\partial^{k} D_{2}(r, t, v)}{\partial r^{k}}\right| d v \\
& +\int_{0}^{\theta}\left|\frac{\partial F_{1}\left(r+D_{2}, v, t+r v+D_{1}\right)}{\partial t}\right| \cdot\left|\frac{\partial^{k} D_{1}(r, t, v)}{\partial r^{k}}\right| d v \\
& +C\left(r^{-\mu_{0}}+\varepsilon\right) \\
& \cdot \sum_{k_{1}+k_{2}=k} \sum_{\substack{i_{1}+\cdots+i_{s}=k_{1} \\
j_{1}+\cdots+j_{v}=k_{2}}}\left|\frac{\partial^{i_{1}}\left(r+D_{2}\right)}{\partial r^{i_{1}}}\right| \cdots\left|\frac{\partial^{i_{s}}\left(r+D_{2}\right)}{\partial r^{i_{s}}}\right| \\
& \times\left|\frac{\partial^{j_{1}}\left(r+D_{1}\right)}{\partial r^{j_{1}}}\right| \cdots\left|\frac{\partial^{j_{\nu}}\left(r+D_{1}\right)}{\partial r^{j_{\nu}}}\right| \\
& \leq\left|\frac{\partial^{k} D_{2}(r, t, \theta)}{\partial r^{k}}\right|+C r^{-1}\left(r^{-\mu_{0}}+\varepsilon\right) \cdot\left|\frac{\partial^{k} D_{2}(r, t, v)}{\partial r^{k}}\right| \\
& +C\left(r^{-\mu_{0}}+\varepsilon\right) \cdot\left|\frac{\partial^{k} D_{1}(r, t, v)}{\partial r^{k}}\right|+C\left(r^{-\mu_{0}}+\varepsilon\right),
\end{aligned}
$$

where $s+v \leq 2$. Hence,

$$
\begin{aligned}
& \left|\frac{\partial^{k} D_{1}(r, t, \theta)}{\partial r^{k}}\right| \leq C\left(r^{-\mu_{0}}+\varepsilon\right), \\
& \left|\frac{\partial^{k} D_{2}(r, t, \theta)}{\partial r^{k}}\right| \leq C\left(r^{-\mu_{0}}+\varepsilon\right) .
\end{aligned}
$$

(3) We can prove that

$$
\begin{aligned}
& \left|\frac{\partial^{m} D_{1}(r, t, \theta)}{\partial t^{m}}\right| \leq C\left(r^{-\mu_{0}}+\varepsilon\right), \\
& \left|\frac{\partial^{m} D_{2}(r, t, \theta)}{\partial t^{m}}\right| \leq C\left(r^{-\mu_{0}}+\varepsilon\right)
\end{aligned}
$$

similarly to (2) when $m \neq 0$.

(4) we have that

$$
\begin{aligned}
& \left|\frac{\partial^{2} D_{2}(r, t, \theta)}{\partial r \partial t}\right| \\
& \leq \int_{0}^{\theta}\left|\frac{\partial F_{2}\left(r+D_{2}, v, t+r v+D_{1}\right)}{\partial r}\right| \cdot\left|\frac{\partial^{2} D_{2}(r, t, \theta)}{\partial r \partial t}\right| d v \\
& +\int_{0}^{\theta}\left|\frac{\partial F_{2}\left(r+D_{2}, v, t+r v+D_{1}\right)}{\partial t}\right| \cdot\left|\frac{\partial^{2} D_{1}(r, t, \theta)}{\partial r \partial t}\right| d v \\
& +C\left(r^{-\mu_{0}}+\varepsilon\right) \\
& \cdot\left(\frac{\partial D_{2}(r, t, \theta)}{\partial r}+\frac{\partial D_{1}(r, t, \theta)}{\partial t}+\frac{\partial D_{1}(r, t, \theta)}{\partial r}\right. \\
& \left.+\frac{\partial D_{2}(r, t, \theta)}{\partial t}+1\right) \\
& +\int_{0}^{1}\left|S_{1}(v)\right| d v \\
& \cdot \int_{0}^{\theta}\left|\frac{\partial F_{3}\left(r+D_{2}, v, t+r v+D_{1}\right)}{\partial r}\right| \cdot\left|\frac{\partial^{2} D_{2}(r, t, \theta)}{\partial r \partial t}\right| d v \\
& +\int_{0}^{1}\left|S_{1}(v)\right| d v \\
& \cdot \int_{0}^{\theta}\left|\frac{\partial F_{3}\left(r+D_{2}, v, t+r v+D_{1}\right)}{\partial t}\right| \cdot\left|\frac{\partial^{2} D_{1}(r, t, \theta)}{\partial r \partial t}\right| d v \\
& \leq C\left(r^{-\mu_{0}}+\varepsilon\right) \cdot\left|\frac{\partial^{2} D_{2}(r, t, \theta)}{\partial r \partial t}\right| \\
& +C\left(r^{-\mu_{0}}+\varepsilon\right) \cdot\left|\frac{\partial^{2} D_{1}(r, t, \theta)}{\partial r \partial t}\right|+C\left(r^{-\mu_{0}}+\varepsilon\right), \\
& \left|\frac{\partial^{2} D_{1}(r, t, \theta)}{\partial r \partial t}\right| \\
& \leq\left|\frac{\partial^{2} D_{2}(r, t, \theta)}{\partial r \partial t}\right|+C\left(r^{-\mu_{0}}+\varepsilon\right) \cdot\left|\frac{\partial^{2} D_{2}(r, t, \theta)}{\partial r \partial t}\right| \\
& +C\left(r^{-\mu_{0}}+\varepsilon\right) \cdot\left|\frac{\partial^{2} D_{1}(r, t, \theta)}{\partial r \partial t}\right|+C\left(r^{-\mu_{0}}+\varepsilon\right) .
\end{aligned}
$$


Hence,

$$
\begin{aligned}
& \left|\frac{\partial^{2} D_{1}(r, t, \theta)}{\partial r \partial t}\right| \leq C\left(r^{-\mu_{0}}+\varepsilon\right), \\
& \left|\frac{\partial^{2} D_{2}(r, t, \theta)}{\partial r \partial t}\right| \leq C\left(r^{-\mu_{0}}+\varepsilon\right) .
\end{aligned}
$$
4.

(5) We can prove (103) similarly to (4) for the left $k+m \leq$

Proof of Boundedness. From Theorem 1.1 in [21] we can see $\Phi^{1}$ possesses a sequence of invariant circles tending to infinity. So, in the original system (13), there exists a corresponding sequence of invariant tori in phase space $(x, \dot{x}, t) \in \mathbb{R}^{2} \times \mathbb{T}$. Then any solution of system (13) is bounded because it must stay within one of those tori.

\section{The Proof of Unboundedness}

In this section, we will prove that all solutions of (8) are unbounded if $B<0$. In this case, $A<0$.

Consider (8) which is equivalent to the following system:

$$
\begin{gathered}
\dot{x}=y, \\
\dot{y}=-A|x|^{\alpha-1} x-f(x) y-\varepsilon \widehat{e}(t)|x|^{\alpha-1} x+p(t) .
\end{gathered}
$$

Replacing (18) by an "auxiliary” system

$$
\begin{gathered}
\dot{x}=y, \\
\dot{y}=A|x|^{\alpha-1} x .
\end{gathered}
$$

Under the transformation (21), the system (113) is transformed into the form

$$
\begin{gathered}
\frac{d \rho}{d t}=-\frac{1}{2-2 b} \rho^{2(1-b)} \widehat{g}^{\prime}(\varphi)+\widehat{h}_{1}(\rho, \varphi, t), \\
\frac{d \varphi}{d t}=\rho^{1-2 b} \hat{g}(\varphi)+\widehat{g}_{1}(\rho, \varphi, t),
\end{gathered}
$$

where

$$
\begin{gathered}
\widehat{g}(\varphi)=(1-b) d+2 b d A\left|S\left(\varphi T_{0}\right)\right|^{\alpha+1} \\
\widehat{h}_{1}(\rho, \varphi, t)=-T_{0} d \rho f\left(\rho^{b} S\left(\varphi T_{0}\right)\right) S^{\prime}\left(\varphi T_{0}\right) C\left(\varphi T_{0}\right) \\
-T_{0} d \varepsilon \rho^{2-2 b}\left|S\left(\varphi T_{0}\right)\right|^{\alpha-1} S\left(\varphi T_{0}\right) S^{\prime}\left(\varphi T_{0}\right) \widehat{e}(t) \\
+T_{0} d \rho^{b} S^{\prime}\left(\varphi T_{0}\right) p(t) \\
\widehat{g}_{1}(\rho, \varphi, t)=b d f\left(\rho^{b} S\left(\varphi T_{0}\right)\right) S\left(\varphi T_{0}\right) C\left(\varphi T_{0}\right) \\
+b d \varepsilon \rho^{1-2 b}\left|S\left(\varphi T_{0}\right)\right|^{\alpha+1} \widehat{e}(t) \\
-b d \rho^{b-1} S\left(\varphi T_{0}\right) p(t) .
\end{gathered}
$$

Thus, the system (115) can be written in the form

$$
\begin{gathered}
\frac{d \rho}{d t}=-\frac{1}{2(1-b)} \hat{g}^{\prime}(\varphi) \rho^{2(1-b)}+O\left(\varepsilon \rho^{2(1-b)}\right), \\
\frac{d \varphi}{d t}=\rho^{1-2 b} \hat{g}(\varphi)+O\left(\varepsilon \rho^{1-2 b}\right) .
\end{gathered}
$$

From the equality

$$
\frac{1}{2} C^{2}(t)+\frac{-A}{\alpha+1}|S(t)|^{\alpha+1}=\frac{1}{2}, \quad \forall t \in \mathbb{R},
$$

it follows that

$$
0 \leq\left|S\left(\varphi T_{0}\right)\right|^{\alpha+1} \leq-\frac{\alpha+1}{2 A} .
$$

Hence, the function $\widehat{g}(\varphi)$ is $C^{1}$, 1-periodic and change the sign. Since $\left|S\left(T_{0}-\varphi T_{0}\right)\right|=\left|S\left(\varphi T_{0}\right)\right|$ for any $\varphi \in[0,1]$, there exists $\varphi_{1} \in(0,1 / 2)$ such that

$$
\left|S\left(T_{0}-\varphi_{1} T_{0}\right)\right|^{\alpha+1}=\left|S\left(\varphi_{1} T_{0}\right)\right|^{\alpha+1}=-\frac{\alpha+1}{4 A} .
$$

That is, $\widehat{g}\left(\varphi_{1}\right)=\widehat{g}\left(1-\varphi_{1}\right)=0$. In view of

$$
S\left(T_{0}-\varphi T_{0}\right)=-S\left(\varphi T_{0}\right), \quad C\left(T_{0}-\varphi T_{0}\right)=C\left(\varphi T_{0}\right),
$$

we find

$$
\begin{aligned}
& \hat{g}^{\prime}\left(\varphi_{1}\right) \cdot \hat{g}^{\prime}\left(1-\varphi_{1}\right) \\
& \quad=-(\alpha+1)^{2}\left(2 b d A T_{0}\right)^{2}\left|S\left(\varphi_{1} T_{0}\right)\right|^{2(\alpha-1)} S^{2}\left(\varphi_{1} T_{0}\right) C^{2}\left(\varphi_{1} T_{0}\right) \\
& \quad<0 .
\end{aligned}
$$

Hence, we obtain that $\hat{g}^{\prime}\left(\varphi_{1}\right)$ or $\hat{g}^{\prime}\left(1-\varphi_{1}\right)$ is negative. This proves that there exists a $\varphi^{*}$ such that $\widehat{g}\left(\varphi^{*}\right)=0$ and $\widehat{g}^{\prime}\left(\varphi^{*}\right)<$ 0 . Therefore, there are $v>0$ and $\delta_{0}>0$ such that $\hat{g}^{\prime}(\varphi)<-\delta_{0}$ for $\varphi \in\left[\varphi^{*}-v, \varphi^{*}+v\right]$ and $\widehat{g}(\varphi)>0$ for $\varphi \in\left(\varphi^{*}-v, \varphi^{*}\right)$, $\widehat{g}(\varphi)<0$ for $\varphi \in\left(\varphi^{*}, \varphi^{*}+v\right)$. Let

$$
\mathscr{K}_{J, v}=\left\{(\rho, \varphi) \in \mathbb{R}^{+} \times \mathbb{T}: \rho>J, \varphi \in\left[\varphi^{*}-v, \varphi^{*}+v\right]\right\} .
$$

Then, if $J$ is sufficiently large, on the set $\mathscr{K}_{J, v}$, we have

$$
\begin{gathered}
-\frac{1}{2(1-b)} \widehat{g}^{\prime}(\varphi) \rho^{2(1-b)}+O\left(\varepsilon \rho^{2(1-b)}\right)>\frac{\delta_{0}}{2} \cdot \rho^{2(1-b)} \\
\rho^{1-2 b} \widehat{g}(\varphi)+O\left(\varepsilon \rho^{1-2 b}\right)>0 \\
\text { for } \rho \geq J, \varphi \in\left[\varphi^{*}-v, \varphi^{*}-\frac{v}{2}\right] \\
\rho^{1-2 b} \hat{g}(\varphi)+O\left(\varepsilon \rho^{1-2 b}\right)<0 \\
\text { for } \rho \geq J, \varphi \in\left[\varphi^{*}+\frac{v}{2}, \varphi^{*}+v\right]
\end{gathered}
$$


From (117) and (124) we obtain, for $t \geq 0$,

$$
\begin{aligned}
& \rho\left(t, \rho_{0}, \varphi_{0}\right) \\
& \quad=\rho_{0}+\int_{0}^{t}\left(-\frac{1}{2(1-b)} \hat{g}^{\prime}(\varphi) \rho^{2(1-b)}+O\left(\varepsilon \rho^{2(1-b)}\right)\right) d t \\
& \quad>\rho_{0}+\int_{0}^{t} \frac{\delta_{0}}{2} \cdot \rho^{2(1-b)} d t \geq \rho_{0}>J .
\end{aligned}
$$

Moreover, for $\rho\left(t, \rho_{0}, \varphi_{0}\right)>J$ and $\varphi\left(t, \rho_{0}, \varphi_{0}\right) \in\left[\varphi^{*}-v, \varphi^{*}-v /\right.$ 2] $\cup\left[\varphi^{*}+v / 2, \varphi^{*}+v\right]$, we have

$$
\begin{aligned}
\rho^{1-2 b} & \widehat{g}(\varphi)+O\left(\varepsilon \rho^{1-2 b}\right) \\
& =\rho^{1-2 b} \widehat{g}^{\prime}(\bar{\varphi})\left(\varphi-\varphi^{*}\right)+O\left(\varepsilon \rho^{1-2 b}\right) \\
& <-\frac{\delta_{0}}{2}\left(\varphi-\varphi^{*}\right) \rho^{1-2 b} .
\end{aligned}
$$

From (126) and (127), it follows that any solution $\left(\rho\left(t, \rho_{0}\right.\right.$, $\left.\left.\varphi_{0}\right), \varphi\left(t, \rho_{0}, \varphi_{0}\right)\right)$ of $(115)$ with the initial condition $\left(\rho\left(0, \rho_{0}\right.\right.$, $\left.\left.\varphi_{0}\right), \varphi\left(0, \rho_{0}, \varphi_{0}\right)\right)=\left(\rho_{0}, \varphi_{0}\right) \in \mathscr{K}_{J, v}$ always stays in $\mathscr{K}_{J, v}$ and satisfies $\rho\left(t, \rho_{0}, \varphi_{0}\right)>\delta t+\rho(0)$ with $\delta=\delta_{0}^{3-2 b} / 2$, for all $t \geq 0$. The proof of Theorem 3 is completed.

\section{The Proof of Theorem 4}

In this section, we will prove Theorem 4 by using the abstract result on the existence of quasi-periodic solutions proved in [24] in the context Aubry-Mather theory for reversible systems. We only need to show that the Poincaré map (92) has the monotone property; that is,

$$
\frac{\partial t_{1}}{\partial r}(r, t)>0
$$

We can get that

$$
\left|\frac{\partial F_{0}(r, \theta)}{\partial r}\right| \leq C r^{-1-(1+\gamma-3 b / 2) /(2 b-1)}
$$

by Lemma 11, and

$$
\left|\frac{\partial Q_{2}(r, t)}{\partial r}\right| \leq r^{-\mu_{0}}+\varepsilon
$$

by Lemma 12 . Then we have

$$
\frac{\partial t_{1}}{\partial r}(r, t)=1+\int_{0}^{1} \frac{\partial F_{0}}{\partial r} d \theta+\frac{\partial Q_{2}}{\partial r} \longrightarrow c_{0}, \quad \text { as } r \longrightarrow+\infty
$$

where $c_{0} \geq 1-\varepsilon$. Therefore, we have

$$
\frac{\partial t_{1}}{\partial r}(r, t)>0
$$

as $r \gg 1$ and $\varepsilon \ll 1$. This proves the validity of (128).

\section{Acknowledgments}

This work was partially supported by the National Natural Science Foundation of China (Grant nos. 11171185, 10871117) and SDNSF (Grant no. ZR2010AM013).

\section{References}

[1] J. Moser, Stable and Random Motions in Dynamical System, Princeton University Press, Princeton, NJ, USA, 1973.

[2] J. E. Littlewood, Some Problems in Real and Complex Analysis, Heath, Lexington, Mass, USA, 1968.

[3] G. R. Morris, "A case of boundedness in Littlewood's problem on oscillatory differential equations," Bulletin of the Australian Mathematical Society, vol. 14, no. 1, pp. 71-93, 1976.

[4] R. Dieckerhoff and E. Zehnder, "Boundedness of solutions via the twist-theorem," Annali della Scuola Normale Superiore di Pisa, vol. 14, no. 1, pp. 79-95, 1987.

[5] S. Laederich and M. Levi, "Invariant curves and time-dependent potentials," Ergodic Theory and Dynamical Systems, vol. 11, no. 2, pp. 365-378, 1991.

[6] M. Levi, "Quasiperiodic motions in superquadratic timeperiodic potentials," Communications in Mathematical Physics, vol. 143, no. 1, pp. 43-83, 1991.

[7] M. Levi, "KAM theory for particles in periodic potentials," Ergodic Theory and Dynamical Systems, vol. 10, no. 4, pp. 777785, 1990.

[8] B. Liu, "Boundedness for solutions of nonlinear Hill's equations with periodic forcing terms via Moser's twist theorem," Journal of Differential Equations, vol. 79, no. 2, pp. 304-315, 1989.

[9] Y. M. Long, "An unbounded solution of a superlinear Duffing's equation," Acta Mathematica Sinica, vol. 7, no. 4, pp. 360-369, 1991.

[10] Y. Wang, "Unboundedness in a Duffing equation with polynomial potentials," Journal of Differential Equations, vol. 160, no. 2, pp. 467-479, 2000.

[11] Y. Wang and J. You, "Boundedness of solutions for polynomial potentials with $C^{2}$ time dependent coefficients," Zeitschrift für Angewandte Mathematik und Physik, vol. 47, no. 6, pp. 943-952, 1996.

[12] J. You, "Boundedness of solutions of super-linear Duffing's equations," Scientia Sinica, vol. 8, pp. 805-817, 1991.

[13] X. Yuan, "Boundedness of solutions for Duffing-type equation," Science in China A, vol. 41, no. 6, pp. 595-605, 1998.

[14] T. Küpper and J. You, "Existence of quasiperiodic solutions and Littlewood's boundedness problem of Duffing equations with subquadratic potentials," Nonlinear Analysis, vol. 35, no. 5, pp. 549-559, 1999.

[15] B. Liu, "On Littlewood's boundedness problem for sublinear Duffing equations," Transactions of the American Mathematical Society, vol. 353, no. 4, pp. 1567-1585, 2001.

[16] R. Ortega and G. Verzini, "A variational method for the existence of bounded solutions of a sublinear forced oscillator," Proceedings of the London Mathematical Society, vol. 88, no. 3, pp. 775-795, 2004.

[17] Y. Wang, "Boundedness for sublinear Duffing equations with time-dependent potentials," Journal of Differential Equations, vol. 247, no. 1, pp. 104-118, 2009.

[18] X. Li, "Boundedness of solutions for sublinear reversible systems," Science in China A, vol. 44, no. 2, pp. 137-144, 2001. 
[19] X. Yang, "Boundedness of solutions for sublinear reversible systems," Applied Mathematics and Computation, vol. 158, no. 2, pp. 389-396, 2004.

[20] X. Wang, "Boundedness for sublinear reversible systems with a nonlinear damping and periodic forcing term," Journal of Mathematical Analysis and Applications, vol. 378, no. 1, pp. 7688, 2011.

[21] M. B. Sevryuk, Reversible Systems, vol. 1211 of Lecture Notes in Mathematics, Springer, Berlin, Germany, 1986.

[22] J. Moser, "Convergent series expansions for quasi-periodic motions," Mathematische Annalen, vol. 169, pp. 136-176, 1967.

[23] J. Moser, Stable and Random Motions in Dynamical Systems, Princeton University Press, Princeton, NJ, USA, 1973.

[24] S.-N. Chow and M. L. Pei, "Aubry-Mather theorem and quasiperiodic orbits for time dependent reversible systems," Nonlinear Analysis, vol. 25, no. 9-10, pp. 905-931, 1995. 


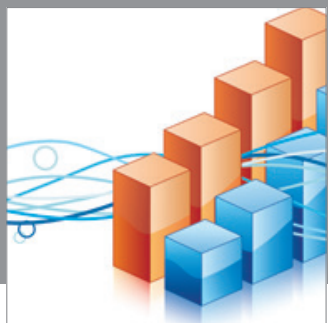

Advances in

Operations Research

mansans

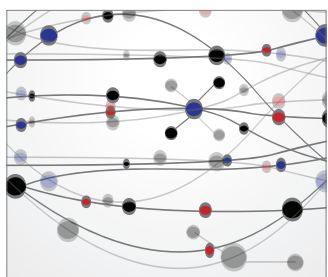

The Scientific World Journal
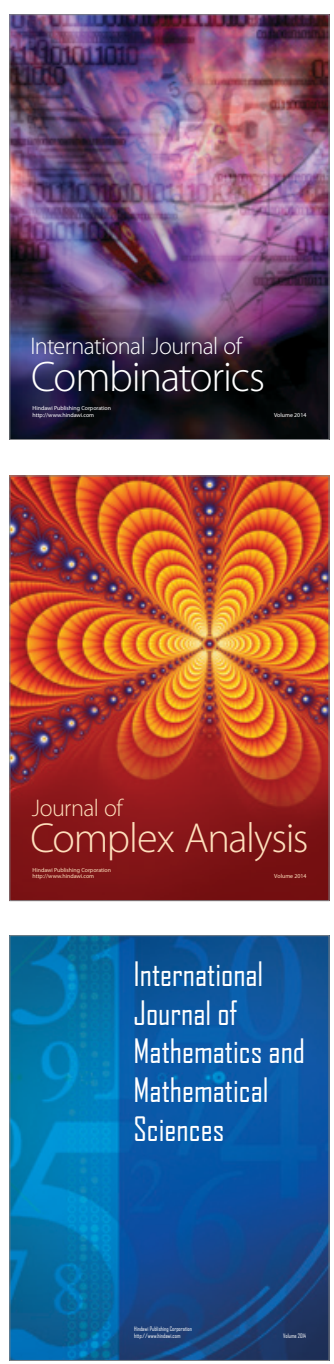
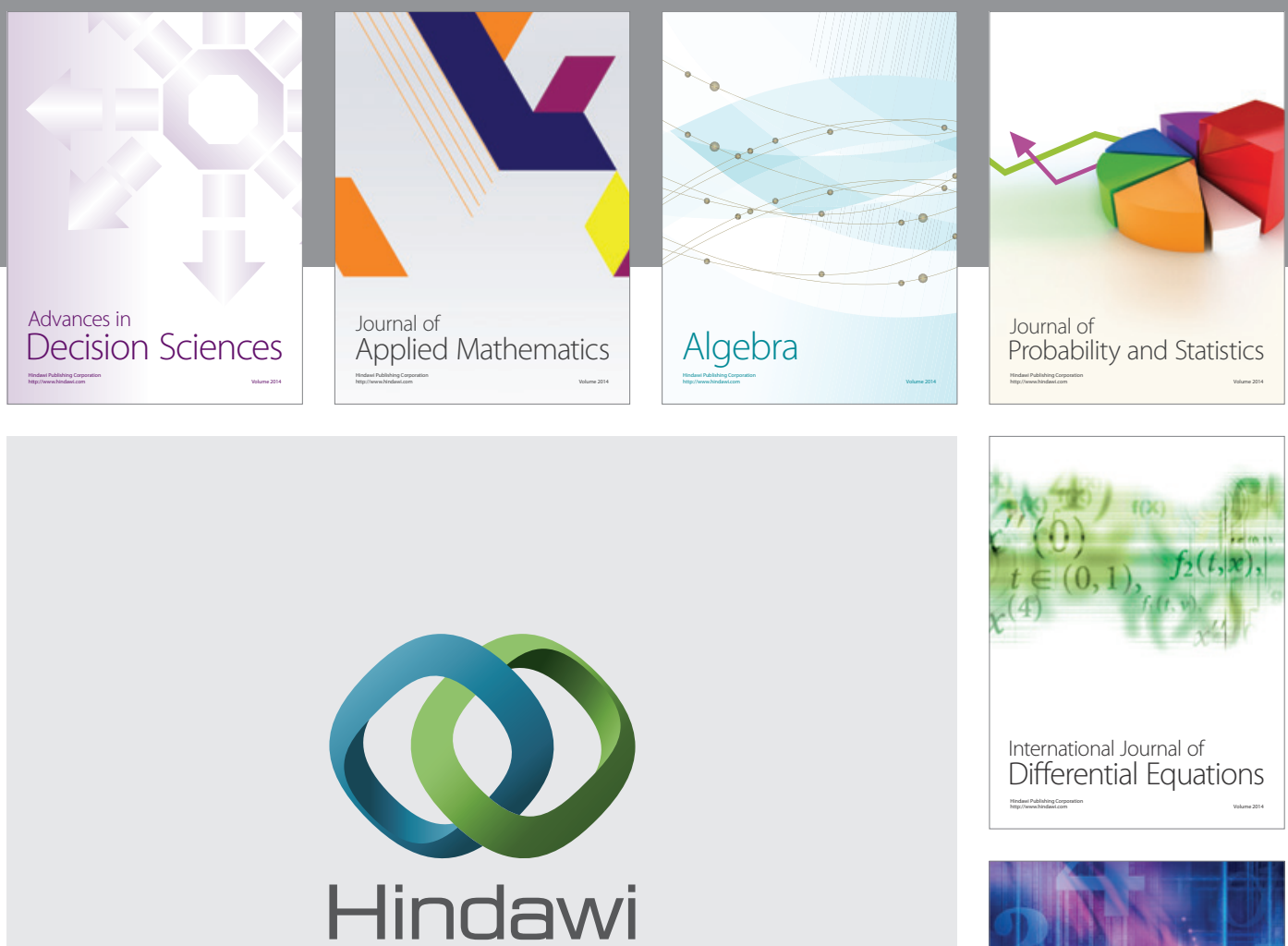

Submit your manuscripts at http://www.hindawi.com
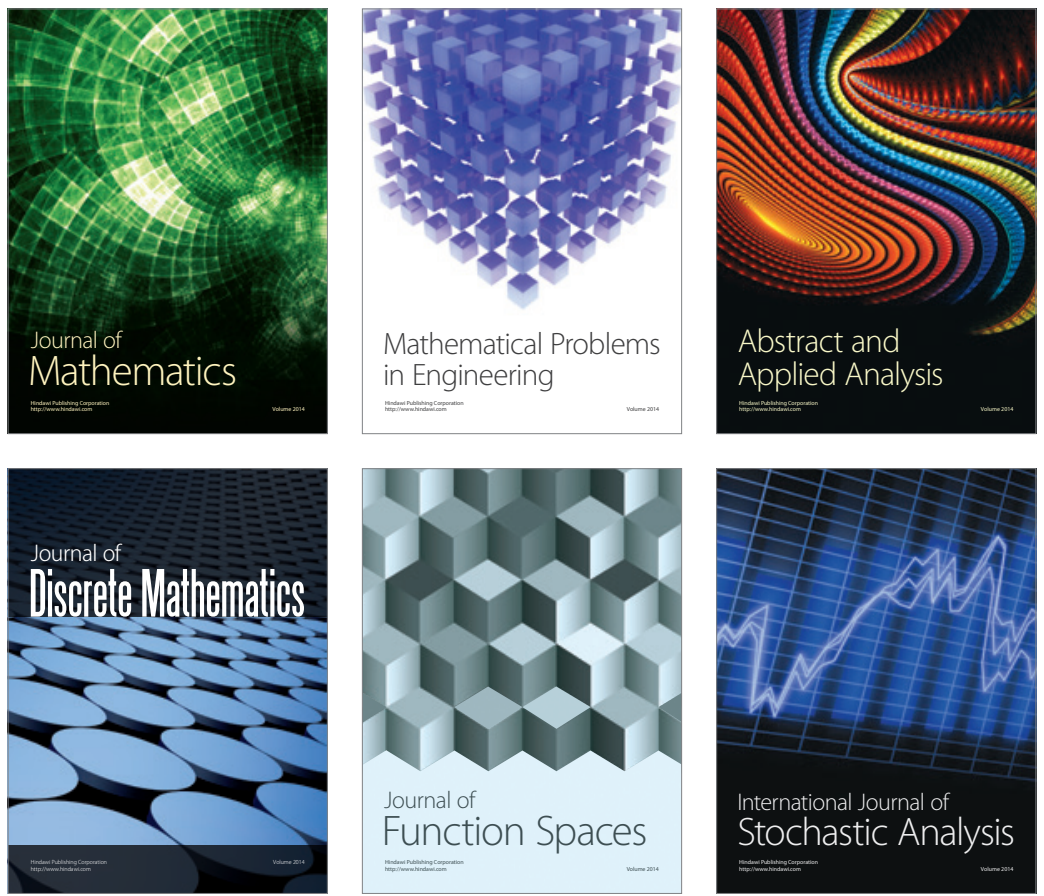

Journal of

Function Spaces

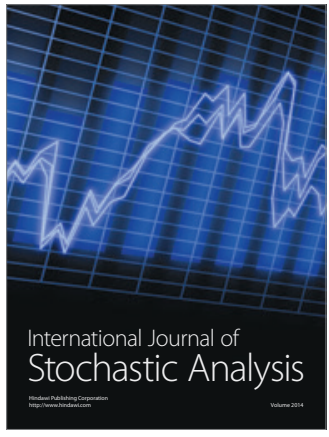

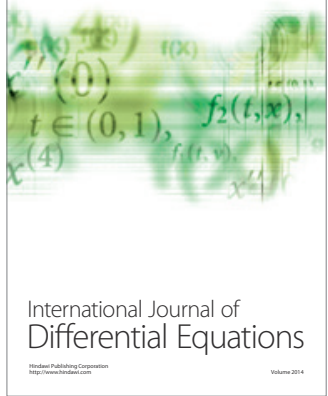
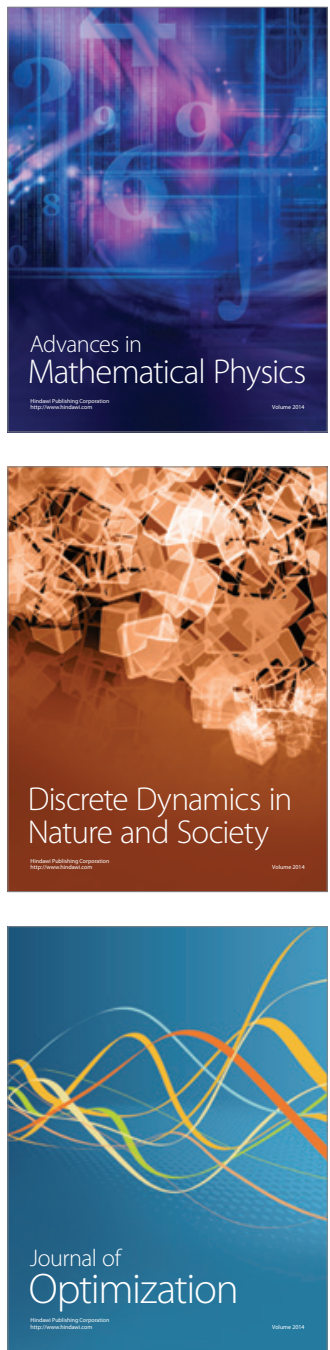